\title{
Language and archeology: some methodological problems. 1. Indo-European and Altaic landscapes
}

\begin{abstract}
The article is the first part of a larger work that represents an attempt to systematize our ideas on the natural environment and material culture of the Proto-Indo-Europeans. It is based on a more or less complete selection of reconstructed words from the appropriate semantic areas and on their comparison with a similar selection performed for a protolanguage of similar time depth, whose speakers evidently inhabited a territory that was not in contact with the Proto-Indo-European one - Proto-Altaic. In this part, only the words that belong to the semantic field of landscape terms are analyzed. The main conclusion is that the hypothesis of a steppe environment is more applicable for the Proto-Altaic population, whereas for Proto-Indo-Europeans a mountainous region seems more appropriate. As for the water bodies, for Proto-Indo-Europeans we should suppose the existence of a sea (or of a very big lake), and for speakers of Proto-Altaic, the existence of very big rivers with season floods.
\end{abstract}

Keywords: Indo-European homeland, Altaic homeland, Wörter und Sachen, semantic reconstruction, proto-lexicon.

In order to reconstruct the phylogenetic tree of a language family, it is essential to understand what the human language is in general, and how the individual languages could be classified as nodes on one phylogenetic tree. Language may be considered a semiotic system that consists of signs and relations between signs, and serves to transmit information within a community; it is independent of individual speakers, and it has the property of changing over time. As a rule, the language sign is a two-sided entity that includes both "semantics" and "text". The link between the semantic side and the textual side is arbitrary, conditioned by the tradition of each individual language. Therefore, any homogeny between the textual sides of the words (morphemes, signs) and the same meanings in two different languages (which is what linguists often observe in practice) needs an explanation. If the homogeny involves large sets of words, random coincidence is statistically unlikely. For this reason, the basic assumption of comparative historical linguistics is that these multiple homogeneous coincidences indicate that any such pair of signs represents two different reflections of one proto-sign.

The matches between the textual sides should not be necessarily exact (literal); most often, two sets of words in two languages can be deduced from a third (hypothetical), "deep", form of the words through the application of regular phonetic rules. These "deep" forms, coupled with their meanings, are considered as proto-signs, which allows for their historic interpretation. Namely, we believe them to have been integral constituents of a proto-language that is reflected in both of the recent languages. But if such a proto-language existed, there must also have been a certain community of speakers that used this proto-language for communication. This raises the question of what kind of people this community included, where it was located and how it functioned. 
It is quite reasonable to try and find any facts from other historical disciplines that could verify the existence of such a community. However, in doing that, one should not forget about the basic meaning of the term "Proto-Indo-Europeans" - a hypothetical ethnos that used to speak the reconstructed Proto-Indo-European language, which is pretty much all that we definitively know about it. Consequently, it is the linguistic part of the information that shall get priority in our research by definition.

Since most of human history lies outside the boundaries of historical records, our only possibility of retrieving lost knowledge lies in the comparison of the results of archeological excavations with the data of our linguistic reconstruction. As far as interdisciplinary cooperation is concerned, this comparison involves two types of problems. The first one is that the correlation between any particular archeological culture and any particular protolanguage cannot be established directly: there is no reason to think that the area occupied by this culture was inhabited at the time by speakers of only one language. Consequently, talking about, e.g., an "Indo-European archeological culture", generally makes little sense. The other part, of a more subjective and technical nature, is that, unfortunately, interaction between these two disciplines is often belated, so that the archeologists build their models on fifty-year-old linguistic data, and vice versa.

However, in some cases we can state that the presumable speakers of a particular protolanguage could (or could not) be the subjects of a particular culture or inhabit a particular area. This can be established by analyzing the reconstructed lexical corpus, with a simple assumption: we expect that if a word that defines, e.g., a 'plough', is reconstructible for a protolanguage, then the speakers of this language could talk about ploughs and, therefore, possess them.

Now the reconstruction of the proto-lexicon involves not only the reconstruction of the phonetic shape of the word or stem, but also the reconstruction of the word's meaning(s). When reconstructing the phonological aspects of the lexical entities, we have strict criteria that help us distinguish between genetically conditioned and typologically conditioned features. In the case of semantic reconstruction, these criteria are far more obscure. In general, linguists are guided by vague ideas of semantic similarity; at best, they rely on typologically similar cases of semantic change that are historically attested for different languages. Thus, the reconstruction of the so-called "world picture" for any proto-ethnos often places the researcher on shaky ground. It is clear that such work (traditionally defined as studies in Wörter und Sachen) should be more productive if the material were to be organized typologically. But any such typology should be founded on compatible data sets. If we produce a "proto-cultural reconstruction" for, e.g., Indo-European, it does not become more convincing by features that reveal any similarity with the "world picture" of "archaic peoples", regardless of whether we are talking of, e.g., the Bushmen (San), the aboriginal Australians, or "of the Shoshonies and Blackfeet". On the contrary - if we do not see any differentiating features, it is highly probable that what we have before us is not a reconstructed "world picture" that is specific for Proto-Indo-Europeans, but a general set of typologically natural archetypes, constructed in accordance with the personal beliefs of the researcher.

For some years already, we have been working on the reconstruction of Proto-Altaic culture, using the reconstructed Proto-Altaic vocabulary. Now that, with the publication of EDAL, we have at our disposal at least two more or less fully and reliably reconstructed protolexicons for two similarly dated proto-languages $\left(6^{\text {th }}-5^{\text {th }}\right.$ mill. вС for Proto-Altaic, $5^{\text {th }}-4^{\text {th }}$ mill. ВС for Proto-Indo-European), it becomes possible to compare these proto-lexicons with particular attention to the semantic areas that are most diagnostic for the proto-homeland and protoculture of both of these hypothetical ethnic groups. 
Of the two, the problem of Indo-European proto-homeland and proto-culture has a long tradition. Today, three hypotheses on the IE proto-homeland are most popular: the Northern Black Sea steppe area (validated in the works of M. Gimbutas and endorsed, among others, by P. Friedrich, D. Q. Adams, J. P. Mallory, D. W. Anthony), the Balkans or Carpatho-Balkan area (validated by a number of Hungarian archeologists and, in Russia, by I. M. Diakonoff, V. A. Safronov), and the Anatolian area (supported by V. V. Ivanov, T. V. Gamkrelidze, C. Renfrew). Since the Proto-Altaic reconstruction is much younger, there is currently but one hypothesis on the Proto-Altaic homeland, suggested by G. Ramstedt and supported by K. H. Menges one that is in agreement with the very name of this language family.

The basic works that are used below, as concerns the Indo-European side of the study, are Гамкрелидзе \& Иванов 1984, Schrader \& Nehring, Benveniste 1970, WP (and the database created by S. L. Nikolaev on the basis of this dictionary ${ }^{1}$ ), Pok., Friedrich 1979, Renfrew 1987, and Adams \& Mallory 1997. The main source on Altaic material is EDAL; apart from that source, we also consult some older works on Altaic linguistics, such as the series of papers by Leningrad scholars, led by V. I. Tsintsius, that analyze fragments of the Common Altaic cultural lexicon (published in such series as ОСААЯ, ИОЭАЯ, АЭ). Сf. also my own paper А. Дыбо 1997.

Reconstruction of semantic features in a proto-language may be formalized if we consider the variability of the meanings of individual reflexations as a kind of polysemy (analogous to polysemy within one language or one small group of closely related languages), and then work with this polysemy by comparing it with the common ways of semantic derivation that are attested in synchronic semantics.

Naturally, our definition of "proto-lexemes" will be restricted to non-derived words ${ }^{2}$ or such derivatives as can be reconstructed for the proto-language and cannot be explained as having been separately derived in some daughter languages after a productive pattern ${ }^{3}$.

Reconstruction of lexical items that are relevant for the proto-culture involves a number of problems concerning the semantic description of the so-called "encyclopedically loaded" semantic fields, or "lexics of concrete lexicon". It should be noted that, when working on the entities of an encyclopedically loaded semantic field, the semantic description that is appropriate for historical studies can be obtained if we divide the semantic features that structure the field (or a lexical microsystem within the field) into "functional" ones and "formal" (or "topo-

${ }^{1}$ http://starling.rinet.ru/cgi-bin/response.cgi?root=config\&morpho=0\&basename $=\backslash$ data $\backslash$ ie $\backslash$ piet\&first $=1$

2 The types of derived words that may, however, be included in the procedure of semantic reconstruction for substantive entities were defined in А. Дыбо 1996: 29; these include diminutives (diminutive affixes are almost always semantically "empty", working almost exclusively as stem-building morphemes), substantivated adjectives and locative names. Such reflexations can usually be identified with primary names.

${ }^{3}$ For this reason, we do not consider such words from Mallory \& Adams 2006 as, e.g., 'mldholeh $a^{-}$'clay' (e.g., OE molde 'sand, dust, soil' [NE mould], Grk málthē 'modelling mixture of wax and pith', Skt mrd- 'clay, loam'), since they are derived (by means of heterogeneous suffixes) from the verb *mel- 'to grind'. The second word from Mallory \& Adams 2006 to denote 'clay' ( ${ }^{*} t k w r e h_{1} y o t->$ OIr crē 'clay', Lat crēta 'chalk', Toch A tukri and Toch B kwriye, both 'clay') is rather a term for pottery material, not for a type of landscape. No PIE landscape term can be seen in the connection between OE swelle 'slope, rise in land' and Toch B șale 'mountain', since, contra Mallory \& Adams 2006, neither of them can be traced back to PIE *swelno- 'slope'. The PToch form, according to Adams 651, can be reconstructed in two ways: a) *șw'äle < PIE *swelo-, probably related to Germanic *swel- 'to swell', which is proposed as the formative stem for OE swelle, but not with the same suffix; b) *s'älwe, from a putative PIE *selwo- and connected with Latin silva 'forest' (with dialectal - $i$ - for -e-). The majority of stems, considered below, can be reconstructed as noun stems (often as root nouns) for PIE, and their suffixal extensions in different languages can be interpreted as adjectival or diminutive ones. 
graphic") ones. The words whose meaning contains "functional" elements are the basic points of the semantic structure of the field, while their "topographic" capacity and types of regular polysemy define the direction of semantic shifts undergone by other words of the field.

As an example, among the different names for 'dwelling' one often finds two types of names for 'house'. Those with a functional value mean not only 'a certain type of building', but also 'locus of the subject'. These words show a regular polysemy: 'house' - 'the housedwellers' - 'the family living in the house'. It is clear that such words are basic for the field, generally more frequent and better revealing the tendencies of semantic evolution within their particular semantic field (such as English house, Russian дом). Other words (such as English cottage, Russian хижина) serve to denote only specific types of buildings and do not have such polysemy. The main problem in reconstructing the semantics of the "encyclopedically loaded" words is to reconstruct the "topographic" features, since the functional features are generally preserved or can be traced in the evolution of the lexical field, while the "topographic" ones may be simply replaced along with changes in the surrounding environment, so that, in order to trace them, we have to use indirect evidence (e.g., one can suggest the presence of a rectangular type of dwelling if the language had a regular polysemy between 'inner angle' and 'a part of the dwelling').

Another point is that it is important not only to choose the "diagnostic" proto-words that are the most relevant ones for the problems involved, but to consider the full scope of available etymological evidence in all thematic fields, which permits us to compose a complete picture of the lifestyle shared by the speakers of the proto-language.

Below I list an example of a group of "proto-words", prepared for the procedure of semantic reconstruction. This is the comparison of two fragments of Proto-Indo-European and Proto-Altaic systems of landscape terms. ${ }^{4}$

\section{Landscape}

\begin{tabular}{|c|c|}
\hline Indo-Eur & Altaic \\
\hline "Earth as place" - "earth & "Earth as place" \\
\hline $\mathrm{PIH} * d g^{\prime} h o m /{ }^{*} d g^{\prime} h e m$ 'earth, soil, territory & PA *nālV 'earth, country': PT *jalay 'open treeless \\
\hline face': Hitt. tēkan 'territory, soil, earth surface', dagan, & place, steppe, glade'; PNM *nalai 'wide, vast'; PTM *nā \\
\hline tagan 'down, to the earth', HLuw takamia & 'earth, dry land, field'; Kor. *nàrá(h) 'country'. \# Nostr.: \\
\hline tijammi id.; OInd. kṣấh, gen. jmáh, kṣmáh 'earth, soil, & Dr. *nēl- 'earth' (DED 2913). \\
\hline habitation place', Avesta $z \bar{\jmath}$, gen. zamo, ac & PA (East) *miot $i$ 'earth, dry land': Kor. ${ }^{*} m u t$ 'dry \\
\hline zami 'earth, soil, territory'; Grk. $\chi \theta \omega$ ',-0 & land'; PJa. *mita ( -u-) ‘earth’. \\
\hline soil, country', Slavic *zem-jí̄; *zem-b 'earth, s & PA (West) *ner-(k)a 'earth, floor': PT *jẹr 'earth as \\
\hline Baltic *̌̌em-i $\tilde{a}$ f. 'earth, so & world, earth surface, territorium'; PNM *žirgi 'litter of \\
\hline m.) 'soil', hum & grass or leaves; doormat'; PNTM *nerke 'earth, world; \\
\hline 'soil, c & place under the $\mathrm{h}$ \\
\hline WH I 654, Buck 16, Klc & CT *bodun 'pe \\
\hline MA 174. \# ? Nostr. ${ }^{*} D V G$ - 'ea & ovince'; PTM *megdi / *m \\
\hline I 342, ОСНЯ 1, 220. & lace, enclosed place, \\
\hline
\end{tabular}

${ }^{4}$ For a detailed overview of problems usually encountered in the semantic reconstruction of landscape terms, seе Толстой 1969, Невская 1977.

The illustrative external cognates are adduced according to references; if any reference lacks, the comparison originates from the Nostratic database made within the Tower of Babel project, mostly by S. A. Starostin, G. S. Starostin, S. L. Nikolaev and me. See http://starling.rinet.ru/cgi-bin/main.cgi?root=config\&morpho=0 


$\frac{\text { Indo-European }}{\mathrm{PIH}{ }^{*} w e d n-/^{*} u d n \text { - 'earth, soil, territory': Hitt. utne- n. }}$
'country, village'; Armenian getin 'soil' Martirosian 2006; Grk. (?) oũ $\delta \alpha \varsigma,-\varepsilon o \varsigma$ n. 'soil, earth surface';

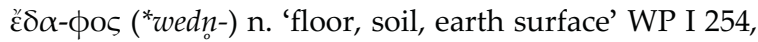
Buck 17-18, Beekes 373.

PIE (Celt-Ital) *têres- 'earth, soil, territory': Latin terra f. 'earth, soil, country, world', terrestris, $-e$ 'placed on the earth', terrēnus, - $a$ 'earthen'; Oskish teer[úm], terúm 'territorium', teras 'terrae'; Celtic *tēros-, *tēres- ? > OIrish tīr n. 'district'; Breton tir 'earth, dry land, soil, country, world' WP I 737, WH II 636, 694, Pok. 1078-1079 (derived from *ters- 'trocknen'). \# Nostr * $t V r$ ' $V$ 'earth, dust', PA *tōr'e 'soil, dust', Kart *mtwwe[r]-.

PIE *mag(')h- 'earth, soil, place': OInd. mahî́ f. 'world, soil'; Celtic *mag- > Gallic Arganto-magus; OIrish mag 'plain earth, unworked field'; Cymr. $m \bar{a}$ 'place' WP II 257, Pok. 709. \# Nostr. *magV 'earth', PA *mūgda, (?) Ur *maje. МССНЯ 342.

PIE *tolH-/*telH-/*tlH-(m)on 'earth, soil, plain place': OInd. talima- n. 'building yard)'; Armenian that 'place, district', thatar 'earthen'; Slavic *tolo, Baltic *tal- $u$ - c. 'floor, bottom, lower part' (OLith. Patulas 'Gott des unterirdischen Reiches', Lith. tilès f. pl. "wooden decking on the bottom of a boat"; OPrus talus 'Fussboden des Zimmers' V. 207; Patollus or Potollos 'Gott des unterirdischen Reiches'); Latin tellūs, gen. -ūris f. 'earth, dry land, soil, country, world'; Celtic OIrish talam, gen. talman 'soil, earth surface, territory' WP I 740, Pok. 1061, MA 174. \# Nostr. *talV 'level ground', PA *t'āle МССНЯ 355.

\section{"Bad earth"}

? PIE (Eur.) *māk(')- 'sandy soil, marsh': Germanic *móx $x-a-\mathrm{m}$. 'sandy soil', Celtic * $m[\bar{a}] k-n i$ - > OIrish mōin 'marsh, peatbog'; OIrish macha 'plain earth' WP II 226 (“unsicher"). Differently in Pok. 699-700. \# Nostr. * $m V K V$ 'hill, bank', Ur. *mäke МССНЯ 371.

\section{Altaic}

yard'; PJa. *mátì 'street, quarter'. \# ? Ur. *mäke 'hill' (МССНЯ 371).

"Earth as soil" - "bad earth" ("sand", "marsh", "salt marsh")

PA *tó̀re 'earth, soil, dust': PCT *tōr 'dust' (OT üzä tuman turdl, asra toz turdl "The fog was hanging above, The dust was rising below"); PNM *tor-tag 'soot, flying dust'; PTung *turV 'earth as soil, territory, world'; Kor. *tìrí 'field, steppe', PJa. *tàra 'dirt'.

PA *siári 'earth, sand; marsh': PT *siař '(salt) marsh'; PM *sirayu 'soil, dust'; PTM *siru- 'sand'; Kor. *hằrk 'earth as soil'; PJa. *situ 'marshland, fen, swampy soil'.

PA *mấro 'sand, cobble-stone soil, marsh': PT *bōr 'soil, clay, chalk'; PNM *mara- 'salt marsh'; PNTung *mar- 'moor, marsh'; Kor. *mòr( $\eta$ ái 'sand', màmằrằ'coarse, cobble-stone soil'; PJa. *mana-n-kua 'sand'.

PA (West) *kiumo 'sand, earth': PT *Kum 'sand'; PM *kumaki 'earth as soil; powders'; PTung *küme 'seashore, beach; barrow'.

PA (West) *kiažurV 'sand, salt marsh, earth': PT *Kajir 'sandy, mellow soil; soil; pebble; pebbly; salt marsh'; PM *kužir 'salt marsh'; PNTung *kužur- 'to cover with ground; to bury'. 


\begin{tabular}{l|l}
\hline \multicolumn{1}{|c|}{ Indo-European } & \multicolumn{1}{c}{ Altaic } \\
\hline & $\begin{array}{l}\text { PA (West) *tap o }(r V \text { ) 'earth as soil, dust': PT *topra-k } \\
\text { 'earth as soil'; PM *toyur- 'soil; dust'; PTung *tap- 'clay; } \\
\text { to soil'. }\end{array}$
\end{tabular}

"Sand - pebble" (+ "sandbank, beach")

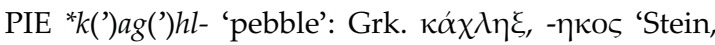
Kiesel' m. 'pebble in river-bed'; Germanic *xagl-a- m., n. 'hail' WP I 338, Pok. 518.

PIE (Eur.) * g'eis- 'gravel, sand': Baltic *̌̌eĩs-r- $r-\hat{\bar{a}}$ f., $-a-$ c., *̌̌is-r-a- c. 'gravel, coarse sand'; Germanic *kís- $a-\mathrm{m}$., n., *kís-il-a- m. 'gravel, sand' WP I 553, Pok. 356. \# Nostr. *kwižzV 'sand': Alt. *kiažurV 'sand, steppe, earth' EDAL 693-694; Ur. * $k O c ̌ V$ 'Sand; sandige Stelle' UEW 226; Kart. Georg. kenç̌- 'pebble' (cf. Georg. kviša, Svan. kwiše 'sand' ?); Drav. SDr *kesar- $\left({ }^{*}-i\right.$-) 'mud, mire' DED 2020. Blažek 1992 135; ND 954, 990a, 1103; A. Dybo 2005.

PIE (GA) *k'ork-/*k'rk- 'pebble': OInd. śarkara- m.,

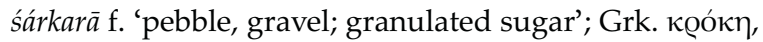

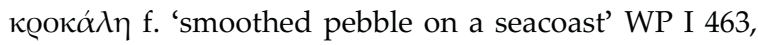
Pok. 615, Buck 51, MA 547.

PIH *pē $n) s-$ 'sand, pebble': Hitt. passila- c. 'pebble', (?) passu-, pissu- 'stone block'; OInd. pāimsú- m., pāimsukan. 'sand, dust'; Avesta pasnu- 'dust, sand'; Slavic *pēs- $z k b$ 'sand' WP II 68, Pok. 824 (deriv. from *pēs- 'blasen'; this could explain sporadic nasalisation but is not quite satisfactory from the semantic point of view), Kloekhorst 650, 652, MA 499.

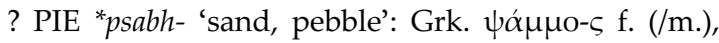
$\psi \alpha \dot{\alpha} \mu \mu-\eta$ f. 'sand', $\psi \tilde{\eta} \phi o-\varsigma$ f. 'pebble'; Latin sabulum, -ī n. 'sand, pebble', sabulō, -ōnis m. 'coarse-grained sand, gravel' WH II 458. Differently in Pok. 145-146, MA 499.

? PIE *samHdh- 'sand': Armenian awaz 'sand'; Grk. ä $\mu \alpha \theta$ o- $\varsigma$ f. 'sand'; Germanic *samd-a- m., f. 'sand' Frisk I 84, Buck 1.215. Differently in Pok. 145-146, MA 499.

\section{“Ore”}

PIE *woHr-/*owHr- 'ore, ore-bearing soil': OInd. vālu$k \bar{a}$-f. 'sand', Germanic *aur-a-n., m.; *ūr-a-n. 'iron sand, ore' Orel 437; Latin urium, - $\bar{\imath}$ n. 'gob'; Celtic OIrish $\bar{u} r$ 'earth, clay'; Tokhar A wāryāñnc, B warañce 'sand, gold dust’ Adams 578, differently Mayr. EWA 2, 547.

\section{Many names for different types of stones: ${ }^{6}$}

$\mathrm{PIH}{ }^{*} h_{2} e k(') h_{2}-$ mon $^{*}{ }^{*} k \bar{a}-$ mon (< ${ }^{*} k e h_{2}-$ mon-) 'stone, rock': Hitt. aku- c. 'stone', akuwant- 'stony'; OInd. áśman'stone, rock; firmament, cloud'; Avesta asman- 'stone, sky', OPers. asman- 'sky', Grk. ök $\mu \omega v$, - ovos m. 'an-

\section{"Sand - pebble" (+ "sandbank, beach")}

PA (East) *̌̌ajk $V$ 'pebble': PTM *̌̌axar(a) 'pebble'; Kor. *čjàkà- 'pebble; mother-of-pearl'.

PA *sájV 'pebble; shallow place': PT *saj ' shallow place with pebbles; arroyo with pebbles; wadi; river'; PNM *sajir 'river-bed, pebble'; PTung *saj- 'sandy mound'; Kor. *săai-m 'spring, shallow well'; PJa. *sái 'sandbank'.

PA *ál'i 'sand, clay': PT *aגu > PCT *ašu 'red clay', PM *ele(r)-sü 'sand, pebble'; PTung *al- 'dirt; bight'; PJa. *ísá-, “ísuá 'shore, coast'.

PA (East) *iฑu 'sandbank': PTM * $(x) i \eta \bar{a}$ 'sand or pebble on the riverbank, sandbank; spit'; Kor. ${ }^{*} j \bar{\partial}$ 'reef, rock in a sea'; PJa. *ía 'bay'.

\section{A single name for stone:}

PA *tiól'ì 'stone': PT *diāl' 'stone'; PM *čilayu 'stone'; PTung *̌̌ola 'stone'; Kor. *tōrh 'stone'; PJa. *(d)ísì 'stone'.

${ }^{5}$ According to MA, the argument against the IE origin in this and many other cases is that "there are comparable forms in non-IE languages, so this is probably a substrate word". Naturally, without any specific hypotheses that speak strongly in favor of borrowing, this argument does not need to be taken into consideration.

${ }^{6}$ See also MA 547-548. 


\begin{tabular}{c|c}
\hline \multicolumn{1}{c|}{ Indo-European } & Altaic \\
\hline vil', Slavic *kāmy, gen. -ene, Baltic *ak-mõ $\left({ }^{*} a k-m e n-e s\right)$ &
\end{tabular}

'stone'; Germanic *xam-ar-a- m.; *xam-al-; *xum-Vl'stone, rock, hammer'; Celtic Gallic acaunum 'rock, cliff, lump, block'. WP I 28, Pok. 18-22. The variability of reflexations could be caused by the contamination of two stems, presumably *akmon/r- 'stone'7 and *k'em-er/n'sky, cloud' (cf. Hitt. kammara- c. 'Wolke, Dunst, Qualm, Rauch' Tischler 472-473, Germ. *xim-in-a-, *xim-il-a- m., Celt. Gael. cwmwl, Bret. koumoul, Corn. comol 'cloud' here rather than borrowed < Lat. cumulus 'heap' [Differently in Pok.]).

PIE *glewH- 'round stone, lump': OInd. glau- m.

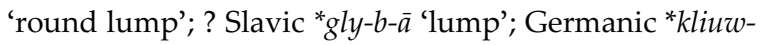
an- m., n., $-\bar{o}(n-)$ f.; *klunj-a- n. 'round stone'; Celtic OIrish glō-snathe, glao-snathe 'plummet'. WP I 612, Pok. 363 (sub *gel- 'round').

? PIE *twrd-/*tword- 'hard stone, quartz; hard as

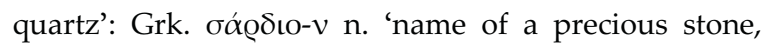
jewel', 'Sarder, Karneol'; Slavic *tvordb(jb) 'hard'; ? Baltic > Lith. tvirta-s 'fest, stark, hart' (acute because of

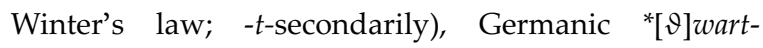
'quartz' WP I 747 (differently in Фасмер and Pok. 1101, Beekes 1308 (derived from $\Sigma \alpha ́ \varrho \delta \varepsilon เ \varsigma)$ ).

? PIE *k'eHil- 'stone, rock, stone flag': OInd. silá $\mathrm{f}$. 'stone, rock, cliff'; Armenian sal 'stone flag, anvil' WP I 454, Pok. 541-542 (from $k^{\prime} \bar{e}(i)-$ : $k^{\prime} \bar{o}(i)-$ : $k^{\prime} \partial(i)$ - 'to sharpen'), Mayr. EWA 2, 640 (“Nicht aufgeklärt”). \# Nostr. *kElV 'stone', Kart *kl-, Drav *kal-.

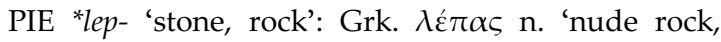
cliff', $\lambda \varepsilon \pi \tilde{\alpha}$ เo- 'rocky, cliffy'; Latin lapis, gen. -idis m. (/f.) 'stone, jevel' (<* $l_{e} p$-ed-s), Umbric abl. vapeře 'throne' WP II 431, WH I 761, Pok. 678, Beekes 848 ("Mediterannean borrowing").

PIE *Hond-/*Hnd- 'stone, rock': OInd. ádri- m. 'stone, rock, mountain'; Celtic *ondes-: MIrish ond, onn, gen. uinde 'stone, rock' Pok. 778, Mayr. EWA I 165, MA 547.

PIE *pels-/*pls- 'stone, rock': OInd. pāṣāṇa- m., pāṣ̂̄ f., pāṣyà- n. 'stone, rock'; Iranian Pashto parș́á 'stone, rock'; Grk. $\pi \varepsilon \dot{\lambda} \lambda \lambda \alpha$ f. 'stone'; Germanic *filz-á- n., *filís-a-; *fulV́s- 'rock'; Celtic *plso- > OIrish all, gen. alle 'rock, cliff' Pok. 807, MA 548, Mayr. EWA 2, 125, Beekes 1168 ("Pre-Greek").

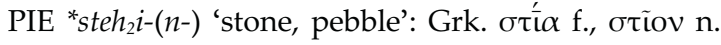
'pebble'; Slavic *stēnā 'stone, rock, wall'; Germanic *stain-a- m. 'stone'. WP II 610 f., Pok. 1010-1011 (as deriv. from stāi- 'to condense, press together', which is semantically unlikely), Beekes 1405.

PIE *g'hwerzd-, 'g'herzdw- 'sharp stone, gravel': Avest.

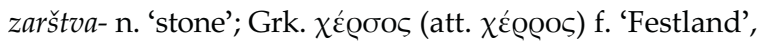

7 The rule of regular depalatalization before resonants in Balt. and Slav. (MA 547) does not work, cf. Slav. ${ }^{*}$ ostrb < ${ }^{*} o s-r-$, Balt. * ${ }^{* c ̌}-r-u$ - id., Lith. šlãpia- 'nass, feucht' etc. 


\begin{tabular}{c|c}
\hline Indo-European & Altaic \\
\hline Slavic ${ }^{*}$ gubrsta, ${ }^{*}$ govrsto, ${ }^{*}$.
\end{tabular}

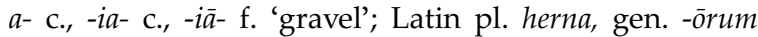
'stones, rocks' (" ${ }^{*}$ 'hers-no-; sabin. nach Serv. Aen.) WH I 643, Beekes 1626, Fraenkel 1328, Pok. 445-446 (as deriv. from *g'her- 'starren', which is semantically unlikely).

PIE *leh ${ }_{1} w-(l \bar{e} u-:$ lau- Pok.) 'stone': Gr. hom. $\lambda \tilde{\alpha} \alpha \varsigma$, Gen. $\lambda \tilde{\alpha} 0 \varsigma$ 'Stein' (Ausgleichung von ursprüngl. ${ }^{*} \lambda \tilde{\eta}_{F} \alpha \varsigma ; \lambda \check{\alpha}_{F} \alpha[\sigma] \mathrm{o} \varsigma$ n.), att. $\lambda \tilde{\alpha} \alpha \varsigma$ und $\lambda \tilde{\alpha} \varsigma \mathrm{m}$. ., Gen. $\lambda \bar{\alpha}$ ov usw.; hom. $\lambda \tilde{\alpha} i \gamma \xi \xi, P l . \lambda \bar{\alpha} i ̈ \gamma \gamma \varepsilon \varsigma$ f. 'Steinchen' (wohl mit Suffixtausch für ${ }^{*} \lambda \bar{\alpha} i ̈ \gamma \kappa-$, vgl. kelt. *līuank-) [Differently by Beekes 817, MA 547: Myc. ra-e-ja 'of

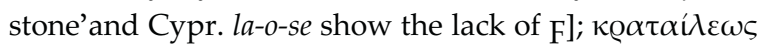

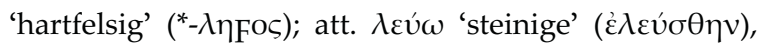
$\lambda \varepsilon v \sigma \tau \eta \dot{0} \varrho$ 'Steiniger' (aus ${ }^{*} \lambda \eta v \sigma-$, idg. *lēus-); ablaut.

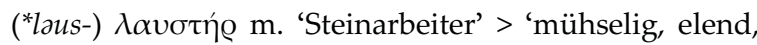
mit Steinen belegter Hausgang'; neben dem $-\alpha \varsigma$-St. ein $-\alpha \varrho-S t .{ }^{*} \lambda \breve{\alpha}_{F} \alpha \varrho$ als Grundlage von att. $\lambda \alpha v ́ \varrho \alpha$, ion.

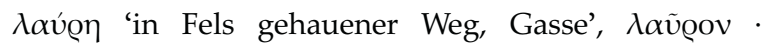

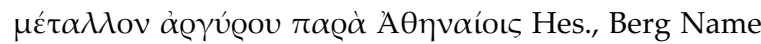

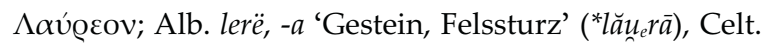
*līuank-, < PIE. *lēuank- 'stone'. WP II 405, Pok. 683, Matasović 242 ( ${ }^{*} l e h_{1} u-s$, Gen. $\left.{ }^{*} l h_{1} w-o s\right)$.

Flat part of relief: "plain earth" - "uncultivated earth" - "free space"

PIE (Eur.) * $k(') a i t o-$ 'forest, uncultivated earth, pasture': Germanic *xái $\vartheta-i-z$ f. 'uncultivated earth, pasture' Orel 154; Latin bü-cētum '(cow) pasture'; Celtic *kayto'wood'. WP I 328 f, Buck 47, Pok. 521, Matasović 198.

PIE (Eur.) *londh-/*Indh- 'free land, heath, steppe': Slavic *lệda, *lệdo, *lẹdjā 'waste ground, clearing overgrown with trees'; Baltic *lind-a- n. 'valley'; Germanic *land-a- n.; 'place, field' Orel 235; Celtic *landā 'open land, pasture, steppe' WP II 438, Pok. 675, Trautmann 157 (from lendh- 'Lende; Niere', semantically unlikely), Matasović 232.

PIE *wen-/*wn- 'outside, forest, field': OInd. vána- $\mathrm{n}$. 'forest, tree'; Avesta van̄a- 'tree', MPers. van, NPers. bun 'tree'; Slavic * $j \bar{o}$ f. 'meadow' WP I 258 (differently in Pok. 1146-1147).

PIE *ghaw- 'space around the village, waste land':

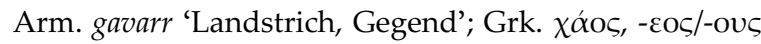
n. 'unbeschränkter Raum, Luftraum; weite Kluft, Schlund'; 'Chaos'; Germ. * 'gau-ja- n., -jō f., -jan- m. 'country, environment' Orel 128. WP I 465, Pok. 449 (as deriv. from $\hat{g} h \bar{e} u-:$ ğhōo $(u)-: \hat{g} h a u-$ 'to yawn, gape' which is sem. unlikely). \# Nostr. PA *kébà( $r V)$ 'field, steppe' 749.
Flat part of relief: hilly steppe. "Plain earth" "steppe" - "eminence"

PA *kéba(rV) 'field, steppe': PM *keyere 'open field, steppe, waste ground; taiga; wlderness'; PNTung *keber'meadow, tundra, plain earth'; PJa. *kápi 'a valley between mountains'

PA (West) *čoli 'steppe': PCT *čöl 'desert, steppe, plain earth (as opposition to qol 'valley' and $d \bar{a} g$ 'mountain'); PNM *čoli-d 'region dotted with lakelets'; PTM *čulbi- 'hill, mound'.

PA (West) *kiàre 'plateau steppe, eminence': PT *Kïr 'plateau, eminence, hilly steppe, desert; a single mountain; mountain top; plain earth; edge, bank'; PM *kira 'mountain ridge'; PTung *xiāri- 'talus, precipice'

\section{"Plain earth" - “open space" - "unpopulated} space"

PA *biogo 'place, open place': PNM *buji- 'far off, unpopulated (place)'; PTM *biga 'field, steppe', PJa. “pía 'room, place; surroundings'.

PA * pàlà 'plain earth': PT *(h)ala- $\eta$ 'open place, glade, meadow, plain earth, hills on the plain'; PTM *pāla-n 'glade, plain place; floor'; Kor. " $p$ òr(h)- 'fields, meadows'; PJa. *pàrà 'field, plain earth, steppe'.

${ }^{8}$ PIE *rowa- 'to open' > 'free space, plain earth': Avesta ravah- 'space, room', ravas-čarāt- 'free'; Germanic *rūm-a- m., *raum-a- m. 'space, room'; Latin rūs, rūris 'country, village, field'; Celtic OIrish rōe, rōi 'plain field' ( ${ }^{*}$ rowesiā), rē 'space, room' ( $\left.{ }^{*} r \bar{e} w i \bar{a}\right)$; Tokhar A, B ru- 'to open'. WP II 356 f., WH II 454, Pok. 874 - this is apparently a group of separate derivatives. 


\begin{tabular}{c|}
\hline Indo-European \\
\hline PIE *
\end{tabular}

PIE *pol-/*pel-/*pl- 'field, plain earth': Armenian hot 'soil, earth, field'; Slavic *pol-j-e; *pol-n-īnā 'field; waste ground'; Germanic *fel- $t-a-n ., m$. Orel 97, *ful- $t-\bar{o}(n-) f$., Orel 117 'plain earth, field, pasture', *fal-ōn- $f$. (> OSwed fala 'Ebene, bes. baumlose; Heide') WP II 61; Pok. 805807 (together with pela-, plā- 'wide, plain'; but these reflexes have no traces of *-H-). \# Nostr. PA *p'àlà 'field, level ground', Drav. *pal- 'plain, valley'.

PIE *dhon-(w-) 'plain earth, wild place': Ind. dhánusn., dhánvan- m., n. 'desert, arid land'; dhánu-, dhanu- $\mathrm{f}$. 'sandbank, sandy shore'; Germ. *dan-jō f., *dan-ja- n., etc. 'den, forest dale'; VLat. danea 'area'. WP I 853, Pok. 249 (together with dhen- 'surface of hand').

\section{Valley}

PIH *Har- 'valley, vale, dale; grotto; swamp': Hitt. hari- c. 'valley'; Armenian ayr 'Spelunke, Grotte'; Baltic *ar-mõ (-men-) c. 'swamp, marsh'. Tischler 172-173.

PIE *ank(')o- 'meadow, valley' Grk. ä $\gamma \kappa o \varsigma$ n. 'Bergschlucht, Felsental'; Germanic *ang-iō f.; *ang-ia- n.; *ang-r-a- m. 'meadow' Orel 19. WP I 60 f, Buck 28.P I 60, Pok. 45-47 (sub ank-, ang- 'to bow').

PIE *dholo-s: Iranian *dara- / darna- 'ravine, valley', Celtic *dolā 'meadow, dale' (Wels dōl 'valley, meadow'), Germ. ${ }^{*} d a l-a-$ n., m., ${ }^{*} d \bar{o} l-,{ }^{*} d a l j-a-$ m., n., ${ }^{*} d a l-j \bar{o}(n-)$, ? *dil-jō(n-) f. (ON dalr 'valley', NE dale, OHG tal, Goth dals, Eng dell (*dhol-yo-), Slav. *dolv (*dhol-u-) 'valley, under side', WP I 864, Pok. 245-246, MA 618, ЭСИЯ II 344-345, Beekes 551, Matasović 103.

PIE *lonko/ā-: Baltic *lanka (Lith. lankà 'valley, rivermeadow', Lett. lañka 'low long flatland'), Slavic *loka 'gulf, valley, meadow, marsh', Preromanian (Celt.?)

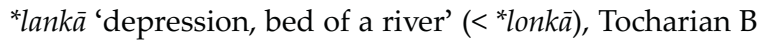
lenke 'valley; cleft'. WP II 435, Trautmann 159, Pok. 676677, Adams 3043, MA 618.

\section{Meadow}

PIE (Eur.) *wongh- 'meadow, field': Baltic *wang-u- c.,

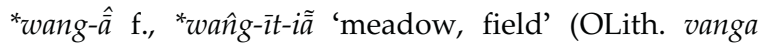
'Acker', Lett. uôdzĩte 'kleiner Bach; sumpfige Stelle im Wald', OPrus. Wangus 'Dammerau'; Kur. > Lett. vañga 'feuchte Wiese mit hohem Gras'); Germanic *wang- $a$ - m. 'meadow, field' Pok. 1149 (Germ., sub ue-n-gh- 'to be bent').

PIE (Celt-Ital) *prāt- 'meadow, hillock': Lat. prātum, $-\bar{\imath}$ n. 'Wiese', Celt. *rāt- > MIr rāth, rāith f. 'Erdwand, Erdbank'; MCymr. bed-rawt 'Grabhügel, Grab', Cymr. beddrod m. 'Grabhügel, Grab', Bret. bez-ret 'Begräbnisplatz, Friedhof'. WH II 358.

PIE *louk- 'woodless field, lawn, glade': OInd. loká$\mathrm{m}$. 'free, open space, world, place'; Baltic *lauk-a- m.

\section{Altaic}

PA (West) *t'äle 'open place': PM *tala 'steppe, open place'; PTM *tālgi- 'far from the shore, open sea; quiet sea surface' \# МССНЯ 355 (PIE *tela-).

\section{Valley}

PA *goblu 'valley': PCT *Kōl 'river valley; ravine'; PM *gowl 'river, river valley; center'; PManch *gola 'middle of river bed, valley between mountains'; Kor. *kōr 'valley'; PJa. *kura 'deep valley' 


\begin{tabular}{|c|c|}
\hline Indo-European & Altaic \\
\hline 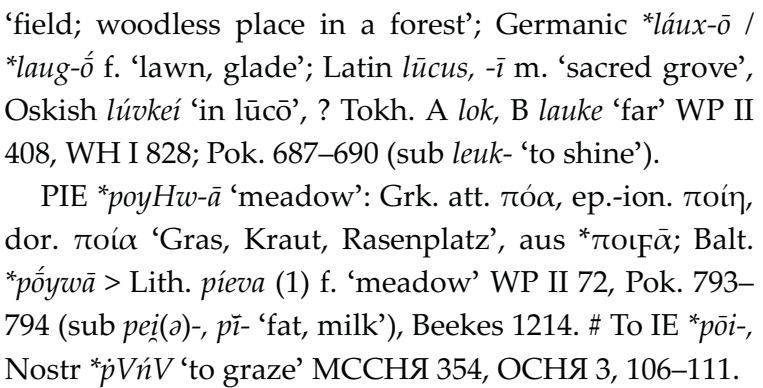 & \\
\hline
\end{tabular}

If we interpret the variability of the meanings as polysemy within a language family, certain definite differences between Altaic and Indo-European may be observed. Thus, it appears that the type of polysemy that is quite familiar for us ("earth as place" - "earth as soil") is characteristic of Indo-European languages, but significantly less so for the Altaic languages, where the meaning "earth as soil" is often connected with such meanings as "bad soil", "marsh", "sand", "salt marsh". On the other hand, the Altaic languages have another series of words meaning "sand", related to the meanings "pebbles" and "shallow place", and this word group has an exact semantic analog in the Indo-European languages. While there is only one name for "stone" in the Altaic languages, we find many names for different types of stones in Indo-European (which brings to mind the well-known story about the lack of a general name for "snow" in Eskimo and the diversity of specific names for different types of snow). Almost obligatorily figuring among the meanings related to the sense of "flat place, plain" in various Altaic groups is the meaning "hill, mound, mountain" - something that would be quite atypical of Indo-European languages. Common words meaning "meadow" as a clearing inside a wood exist in PIE but are absent in PA. This means that the ideas of the corresponding landscape objects must have been significantly different for speakers of Proto-Altaic and Proto-Indo-European.

Let us now try to demonstrate, as completely as possible, the sets of landscape terms that are reconstructible for PIE and for PA.

\section{Mountainous terrain}

\begin{tabular}{|c|c|}
\hline Indo-European & Altaic \\
\hline Mountains & Cliffs \\
\hline PIE ${ }^{*} g^{w} h_{1} r-{ }^{*} g^{w} h_{1}$ or- 'mountain': PIIr *gari-, Ind. girí- & PA (West) *kadV 'rock': PCT *K(i)aja 'rock'; PM *kada \\
\hline 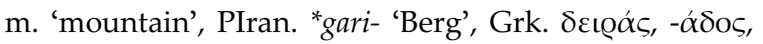 & 'rock'; PTM *kada- 'rock'. \\
\hline 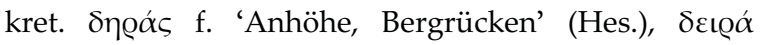 & PA (West) *bajV 'rock': PCT *bAjir 'hill, foot-hill; out- \\
\hline 'mountain range' (Pind.) $\left(<{ }^{*} g^{w} h_{1} r-y a-\right)$; $\beta$ o@é $\bar{\alpha} \varsigma$ 'Nord- & ness'; PNM `baji-ča 'rock'; PNTung *baj- 'rock, cliff' \\
\hline wind' (<* ${ }^{*}{ }^{w} h_{1}$ or- $)$, Alb. gur 'Felsen, Stein' ( $\left.{ }^{*} g^{w}{ }_{e} r i-\right)$; Slav. & PA (East) *p'äko 'rock, cliff': PSTung *pākta 'hill, \\
\hline${ }^{*} g o r \bar{a}\left({ }^{*} g^{w} \partial r-\bar{a}\right)$ 'mountain > mountain forest', Balt. ${ }^{*} g u r-$ & mound; precipice; sand bank'; ? PKor *pàhói 'rock'; PJa \\
\hline$a->$ Lith. gùra-s 'Bergvorsprung', *gir-ja 'forest, tree' WP & ‘paki 'steep rock'. \\
\hline I 682, Pok. 477-478, Buck 25, 48, MA 270, Beekes 227, & \\
\hline 311, ЭСИЯ III, 191-192. \# Nostr. * $g^{w} V r V$ 'mountain, hill', & Hills, slopes \\
\hline PA *kuri, Ur *kur3, Kart *gora, Drav *kur- $\left({ }^{*}-\underline{d}-\right)$. & PA *tújpè ‘top of a mountain': PT *dẹpö 'hill, top'; PM \\
\hline Старостин 2007, 804. & *dobu / *döbe 'hill'; PTM *düj- ( *düb-) 'shore; mountain \\
\hline ? PIE *mon- $(t i-)$ 'mountain': PIran. *mnti- > Av. mati & top; taiga region'; PJa. *(d)ípà 'rock, cliff'. \\
\hline
\end{tabular}




Indo-European

Gebirge', Celt. *monijo- > OIr -monid; Cymr mynydd, Corn meneth, Bret menez 'Berg' WP II 263, Pok. 726 (sub *men- 'to tower'), MA 270, Matasović 277.

$\mathrm{PIH}$ *peru-(n-) 'mountain top': Hitt. peru-na- (piruna-) c. 'Fels' (Friedrich 167), perunant- 'rocky', pirwa- 'bestimmte Art von Felsen (auch als Gottheiten)' (Friedrich 170); Ind. párvata- m., parvatí-, parvatí f. 'mountain, hill, rock'; paru- m. 'mountain', Av. paurvatā- 'mountain'; Tokh. ? A pārem 'rock, stone'. MA 547. \# Nostr. * $\dot{p} V r V$ 'mountain, top', PA *p'óre 'top, mountain top', Kart Georg. prialo 'step rock', Drav *par- 'pebble, gravel';

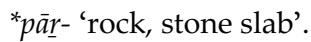

PIE (Eur.) *kaln- 'narrow passage': Slav. *koln-bcb 'ravine, narrow passage between mountains'; Lat. callis, -is m. 'schmaler Bergpfad, Triftweg, Gebirgstriften'. WP I 356, WH I 140, Pok. 524, ЭССЯ 10, 140.

PIE *geHup-/*gHup- 'cave, den, mines': Grk. $\gamma \underline{\text { v́}} \pi \eta \mathrm{f}$. 'den, vulture's nest' (Hes.), Slav. *žúpa 'salt mine; grave'; Germ. *kuf-ēn-/*kōf-ēn- m. 'cove, cave' Orel 222. WP I 555, Фасмер 2, 65-66, Beekes 292 ("European substrate words”). \# Nostr *kop'a 'hole, empty', PA *kóbú 'hollow, cavity', Ur *koppa, Kart *kwab-. ОСНЯ 1, 232233, МССНЯ 358.

$\mathrm{PIH}$ *kolHn-, *kolHm- 'top, hill, rock': Hitt. kalmara-,

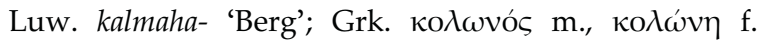

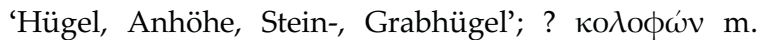
'Gipfel, Spitze, Höhepunkt' statt * $\kappa$ $\lambda \alpha \phi \omega \nu$ auf Grund

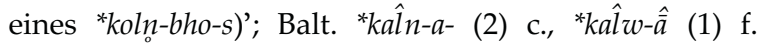
'mountain'; Germ. *xall-u-c., *xull-i- c.; *xulm-a- m., -anm. 'stone, rock' Orel 157; Ital. Lat collis, -is, abl. colle/colli m. 'Hügel, Anhöhe'; columen, (jünger) culmen, -inis n. 'Höhepunkt, Gipfel, First' [Celt. *klukā: OIr., Ir., Gael. cloch 'stone, rock', - non-IE borrowing in Matasović 210]. WP I 433, WH I 197, Pok. 544, Buck 23-24, Beekes 742, MA 270 (as deriv. from *kelh ${ }^{-}$'rise, stand').

\section{Hills, slopes}

? PIE (PGA) *tung w - 'hillock': Ind. tuiga- m. 'elevation, height, mountain'; tunga- 'prominent, lofty, high' Mayr. KEWA 1, 508 ("Nicht überzeugend erklärt”); Grk. $\tau u ́ \mu \beta o-\varsigma \mathrm{m}$. 'mound, burial mound, grave' Beekes 1517 ("Pre-Greek/Mediterannean word" because of Corcyr. $\tau \bar{u} \mu$ os). WP I 706. Or Greek = Lat. tumulus 'earth-hill', Arm. tumb 'landfill, earthen wall', Celt. *tumbo- 'excrescence, hill' (Matasović 394); if so, PIE *tum-bh-, not related with Ind. See Pok. 1072 (all from ${ }^{*} t e H w$ - 'to swell').

PIE * $k($ ')onHm-/k(')neHm- 'slope, mountain forest':

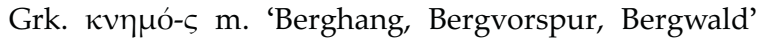
Beekes 723; Germ. *xamm- $a-\mathrm{m}$. 'mountain forest, fenced land' WP I 460, Pok. 613-614.
Altaic

PA (East) *tì̀du 'elevation': PTM *dìdü ( 亏̌) 'mountain ridge'; PKor *tùtán 'hill, elevation'; PJa. *tùtùmí 'dike'.

PA (West) *dión(š)e 'slope, bank': (?) PT *jān 'side', PNM *denž $i$ 'terrace (between the steppe and the river bank)', PTung *dunse 'dry land, coast; wood, taiga'.

PA (East) *anta $(g V)$ 'hill, slope': PTM *antaga 'slope of a mountain'; PKor *antak(h) 'hill'; PJa. *antuma 'East' \# PIE *ant- (MCCHЯ 354).

PA biòsá-gV 'woodless mountain slope': CT *basig 'field'; PNTung *bosoga 'northern slope'; PJa *bàsái 'early rice'.

PA *biuge 'slope, hill': ? PT > Oghuz *bögür 'mountain slope'; PNM *böyerüg 'mountain slopes, hill'; PTung *buga(n) 'hill, mound'; ? PKor *pàhói 'rock'; PJa *ba 'hill, hillock'.

PA *kuri 'hill, cliff': PCT *Korum 'rock, cliff, heap of stones'; PNM *kür 'precipice, rock'; PTM *xurē 'mountain, rock'; PKor *kòrán 'embankment, boundary'; PJa *kùrùa 'dike, boundary'. \# PIE * ${ }^{\text {wer }}$ er- (WP 1, 682).

PA (West) *sira 'hill, high mountain': PT *sirt 'mountain ridge'; PNM *siro- 'rock, high mountain'; PTung *sirk- 'small hillock, cape'.

PA *úkè 'hill': PNM *(h)ukara 'hill'; PTM *(x)uKu'hill'; PJa *báká 'hill'.

PA (West) *î́ńa 'pit, ravine': PT *ījn 'pit, lair'; PNM *oni 'defile, gorge'; PSTung *uńi 'small river, brook'.

\section{To cross mountains}

PA *āl'a 'to cross (a mountain)': PCT *(i) $\bar{a} \lambda$ - 'to cross (a mountain); to surpass'; PM *alu- 'on the other side; far away'; PTM *ala- 'to cross (a mountain); mountain pass'; PJa *asu 'steep bank, precipice'. \# PIE *al- 'other side’ (МССНЯ 372, ОСНЯ 1, 274-275).

PA *dāpa 'to cross (a mountain)': PM *daba- 'cross (a mountain)'; PTM *dāb- 'to cross (a river)'; PJa *dàmà 'mountain'. 


\begin{tabular}{|c|c|}
\hline Indo-European & Altaic \\
\hline 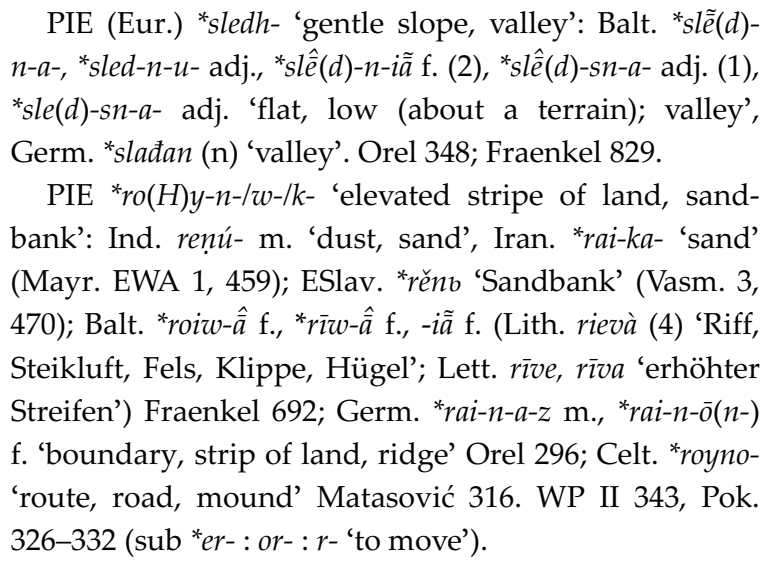 & \\
\hline
\end{tabular}

\section{Water landscape}

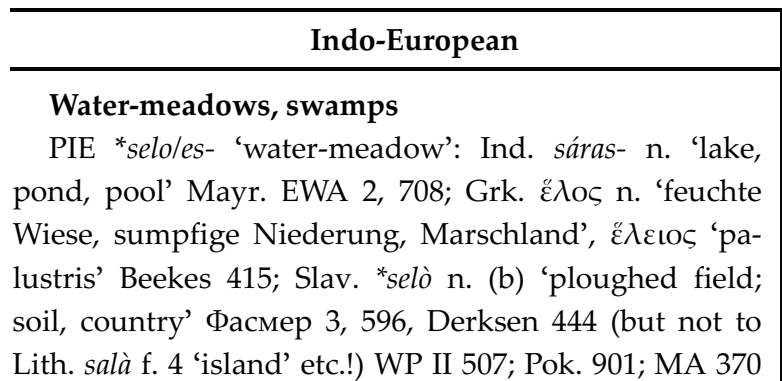

(+ Wels heledd 'meadow along the river' < *sel-iyā).

PIE *pa(H)lw- 'clay, mud, morass': Ind. palvala- n. 'pool, small tank, pond' Mayr. EWA 2, 104; Grk. $\pi \eta \lambda$ ’ó Hes., Dor. $\pi \alpha \lambda$ ó $\varsigma$ m. 'Lehm, Ton, Schlamm, Kot, Morast' Beekes 1186 (“without convincing etymology”); Balt. *pal-ia- f. 'Sumpf, Moor' Fraenkel 1, 532; Lat. palūs, gen. -üdis f. 'stehendes Wasser, Sumpf, Pfütze' WH 2, 243 (Alb. püł 'Wald' borrowed from Balk.-Rom. * padūlem < Lat. palūdem, Orel AlbD 353). WP II 55, Pok. 798-801 (sub *pela- 'full') \# Nostr. * $\dot{p} V l V$ 'wash, flow', PA *póle 'wet, succulent; grass, plant', Ur. * pülk3.

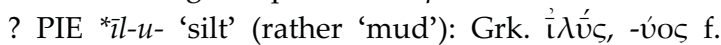
'Schlamm, Kott, Morast' Beekes 589; Slav. *jílto 'bog, silt, mud' Derksen 211 (*jolv); Balt. *î̀ l-u- (1) adj. > Lett. îls 'very dark'. WP I 163, Pok. 499, MA 370-371. \# Nostr. *néelV 'earth': PA *nāll 'earth, land'; Drav. *nél- 'earth' (DED 2913). А. Дыбо 2000.

PIE (Eur.) *balH- 'marsh': Slav. *bol-nb-je n. (a) 'low

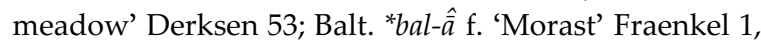
30, Germ. *pōl-az 'pool' Falk \& Torp 151, Orel 293 (Celt. Ir poll, pull; Cymr pwll < Germ.?). WP II 176 \# Nostr. *palV 'swamp, marsh', PA *biălu 'dirt, mud'. МССНЯ 331, ОСНЯ 2, 97-98.

PIE (Eur.) ‘bholHt-om 'marsh': Slav. *bólto n. (a) 'swamp' Derksen 53; Balt. OPrus. *balt-an 'marsh' (in 


\section{$\frac{\text { Indo-European }}{\text { toponyms, Топоров 1, 189, Mažiulis 1, 132); \{Lett. Balates }}$} kalns 'Sumpfberg' < Slav.\}; Germ. *puld-r m.: ONord. poll-r m. 'runde Bucht, Teich', MDutch polre, polder $\mathrm{m} ., \mathrm{f}$. 'polder; dijk'; Dutch polder 'Marschland' (> EFris. polder 'Marschland') De Vries 427; Alb. baltë f. (<*baltā, NPl neu.), Balt. m. 'swamp' (> Rum. baltă 'swamp', Middle Greek $\beta \alpha ́ \lambda \tau o \varsigma)$ Orel AlbD 15-16; near can be Illyr. *balta 'Sumpf', Lat. blatea f., 'Kotklümpchen', ODalm. balta 'Sumpfsee'. WP II 176, Pok. 118-120 (mixed).

PIE (Eur.) *k(')woin- 'marsh': Germ. *xwain-ō 'swampy field' Orel 197, *xwin- id. Falk \& Torp 86; Lat. caenum, $-\vec{\imath}$ n. 'Schmutz, Schlamm, Kot, Unflat' WH 1, 132; Celt. OIr. cōennach 'Moos' WP I 469, Pok. 628 (sub k'uei- 'dirt').

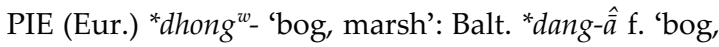
marsh' (> Lett. dañga 'kotige Pfütze, weiches morastiges Land', length because of Winter's law); Germ. đank(w)-ō f., 'bog' Orel 68. WP I 851, Pok. 247-248 (sub *dhema- 'to smoke').

PIE (Eur.) *pan- 'mud, slush, morass': Balt. *pan-iã f. > OPrus. pannean 'Moorbruch' Mažiulis 3, 217; Germ. *fan-ja-n n. 'fen, marsh, mud' Orel 92; Celt. MIr an 'Wasser', enach 'swamp'; Gaul anam 'paludem'. WP II 5, Pok. 807-808, MA 370-371.

\section{Shore}

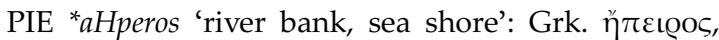

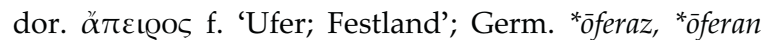
'bank, shore' Orel 290; Arm. ap'n 'shore'. WP I 48, Pok. 53, MA 343.

? PIE (GA) *dhisn- 'sand-hill, dune': Ind. dhíṣyya- m. 'a heap of earth covered with sand on which the fire is placed' Mayr. KEWA 2, 103, Mayr. EWA 1, 792; Grk. $\theta^{\prime} \bar{\imath} \varsigma$, gen. $\theta^{\top} \mathbf{\imath}$ ós (*dhisn-s, *dhisn-os, see Sihler 216) m./f. 'Haufen; Sandhaufen (am Meere), Düne, Gestade' Beekes 596 (“Without explanation”). WP I 835 f (differently in Pok.).

PIE (Eur.) *dhūn- 'coast, (dry) land': Balt. *dūn-ia- c.,

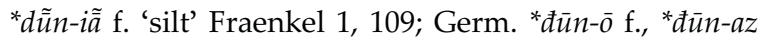
m. 'hill, dune' Orel 80. Pok. 263 (sub *dheu- 'to blow') \# Nostr. * $d V w n V$ 'shore, land', PA *dióna.

\section{Water, wave}

$\mathrm{PIH}$ *weHr-/uHr- 'water, moisture': Luv. wār- 'water' (wārsa is a form of nom.-acc. sg.) Melchert CLL 257; Ind. varī f. pl. 'streams, rivers'; vār, vāri n. 'water'; Avest. vairi- m. 'See'; vār- 'Regen', Pers. bārān 'rain'; Arm. gayrr 'Sumpf, Schlamm'; OPrus wurs < *ūras 'Teich' Mažiulis III 271; Germ. *warōn, *waraz m. 'liquid, water; sea'; Orel 451; Celt. ? MIr feraim 'giesse', ferad 'Feuchtigkeit'; Cymr gweren 'liquamen'; Tokh. *wär 'Wasser' (Adams 577). WP I 268, Buck 3, 45, Pok. 80, MA 636. Probably it
Altaic

\section{Island}

PA *bùke 'tall island': PCT *bük 'wood/hill/meadow on the river bank'; PM *buka 'hillock, canal'; PTM *būKa 'island'; PKor *puk 'heaping of earth'; ? PJa *boka 'hill'.

PA *šiumi 'island; forest': PCT *simek 'forest on the river bank'; PTung *šumi 'foreland, shallow place; tussock'; PKor *sjōm 'island'; PJa *simà 'island'. \# Nostr. *swajmV 'marsh': Ur. FU *śajmz 'Vertiefung, Senkung (mit einem Teich od. Bach)' UEW 457. A. Dybo 2005.

PA *siumyu 'island, shallow place': PNM *sinara- 'island; bend of river'; PTung *sumpi 'tussock (in a swamp)'; PJa *súná 'sand'.

\section{Shore}

PA *bióro 'bank, rift': PCM *borgija 'river rift; hill, mound'; PTM *bir[u]-kan 'precipice; mountain'; PKor *pirá 'bank'.

PA *giru 'shore; road': PT *Kirgak 'shore, edge'; PTM *giri 'shore, riverbed'; PKor *kírh 'road'.

PA *p'ire 'bank, steep bank': PM *her-gi 'steep bank'; PNTung *piri 'steep (slope, bank)'; PJa *pi(n)ti-pa 'bank'.

PA *sap i 'shore': PCT *sep 'duct, river branch, bay'; PSTung *sapsV 'bank, shore'; PJa *sìp̀̀ 'tide'.

\section{Water, wave}

PA *mì̀ri 'water': PM *mören 'river'; PTM *mū 'water'; PKor *mír 'water'; PJa *mí(-n-tú) 'water'.

PA (West) *siuba 'water': PT *sib 'water'; PM *usu 'water'. \# PIE *sew(ə)-, PK *św- (МСCHЯ 341).

PA (East) *lát'á 'wave': PSTung *lāta 'wave, storm'; PJa *nàntá 'open sea'.

PA (West) *čalu 'wave, to overflow': PCT > Oghuz *dalga 'wave'; PM *dolgi-gan 'wave'; PNTung *žal- 'to overflow, to wave'. \# PIE *sol- 'to flow'. 


\begin{tabular}{c|c}
\hline Indo-European & Altaic \\
\hline
\end{tabular}

should be distinguished from ? PIH *HewH- 'to be wet': Hitt. heu- 'Regen' (Tischler 238); hu-r-nai-/-ija- (I), hu-r$n u$ - (I) 'besprengen, befeuchten', Pal. huwarninai 'besprengt' (Tischler 305-306); Ind. avațá-, avatá- m. (*ew-n$t$-) 'a hole, vacuity in the ground', aváni- f. 'stream, river, bed of a river'; Grk. àvavoos 'wasserlos, von Bächen' Balt.: Lith. jáura, pl. -ōs (1) 'Moorgrund, Sumpfland' Fraenkel 1, 198; Germ. *au-r-a-z m. 'wet soil; ocean' Orel 29, ‘ $\bar{u}-r-a-n$ n. 'drizzling rain, mist, fog' Orel 437, Lat. ūrīnārī 'tauchen unter Wasser', ūrīnātor 'Taucher'. WP I 268 f, Buck 37, 45, Pok. 78-81. MA 636 (iuHr-, Balt. + Thrak. iuras 'name of a river') or MA 539 (Ind. avata + Latv. avuots 'spring').

$\mathrm{PIH}$ *we(n)dh- 'water, wave'9 Friedrich 249-250, Adams 511, WP I 252, Pok. 80, MA 636 \# Nostr. PA *udV 'rain', Ur. wete, Drav. *jēed- 'water' МССНЯ 334. Or to PA *untu 'whirlpool, tide'?

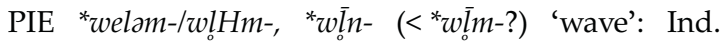
ūrmí- m., f. 'wave, billow'; Avest. varami- 'Woge, Welle'; Slav. *vblnā, Balt. *wiln-i- (2), -jîे f. 'wave'; Germ. *walmi$z$ 'well; boiling'; *wello- f. (<*welna), *walla-z m. 'wave' Falk \& Torp 269, Orel 444; ? Tokh. B yolme 'pond, pool' (Adams 513 with doubts); ? B lāñe 'flood' (Adams 547). WP I 298, Pok. 1140-1142, MA 637 (uncertain).

?? PIE *bhang- 'wave': Ind. bhanga- m. 'wave' (Mayr. KEWA 2, 461, classic Sanskrit, = bhangá- 'das Zerbre-

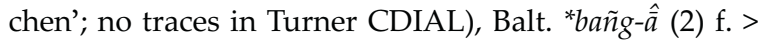
Lith. bangà 'Welle, Woge, Regenguss' WP II $149 \mathrm{f}$

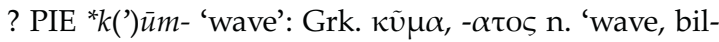
low' Beekes 848; Germ. *xúm-a-n> ONorse hūm n. 'See, Meer' (poet.) n. De Vries 266 (or to hūm 'Dunkelkeit'), Holthausen 132; ? Lat. cumulus, - $\bar{\imath}$ 'Haufe; Menge; Höhepunkt' (cf. Ov. cumulus...immānis aquārum) WH 1, 307. WP I 365, Pok. 592-594 (sub *k'eua- 'to swell') \# Nostr * $k U m V$ 'sand, thin snow': PA *kiumo, Ur. kum 3 МССНЯ 362.

\section{To flow}

PIE (GA) * $d g^{w}$ her - 'to flow, to flood': Ind. kṣárati 'to flow, stream; to melt away, perish', kșara- 'melting away, perishable'; n. (L) 'water'; PIran. *gžar-/xšar- 'to flow, to pour' ЭСИЯ 3, 295-296; Arm. jur, Gen. jroy

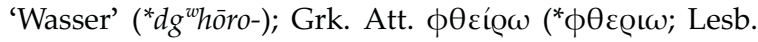

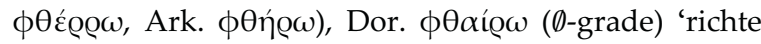
zugrunde'; $\phi \theta$ o@ $\frac{\bar{\alpha}}{\alpha}$ 'Verderben, Vernichtung'; 'Vermis-

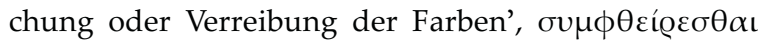
'zusammenströmen'; see also Beekes 1569-1570. WP I 700, \# Nostr *tUKV 'to flow, pour': PA *tiuke, Kart. ${ }^{*}$ tkor-?

\section{To flow}

PA *iàk i 'to flow; basin': PT *iak- 'to flow'; PTM *iaKu 'swamp'; PJa *ikà-i 'pond'.

PA *giàru 'wave, stream': PNM *gori-ka 'small river, rivulet, stream'; PTung *guru- $k i$ 'reach (of river); whirlpool'; PKor *kjár 'wave'.

PA *ùntu 'whirlpool': PNM *undu- 'to burst, whirl (of water); fountain, well'; PTung *onda- 'to rise (of water); water'; PJa *ùntu 'whirlpool'.

PA *čurka 'swift stream, current': PNM *dargil 'rapid current'; PNTung *̌̌urku 'rapid, swift stream; fairway'; PJa *taki 'swift current, waterfall'

${ }^{9}$ For a new interpretation of the traditionally reconstructed set of roots *wed-, *wet-, *und-, see V. Dybo 2002, 413-415, 468. 


\begin{tabular}{c|c}
\hline Indo-European & \\
\hline
\end{tabular}

PIE (Eur.) *sol- 'to flow > island': ? Ind. salilám 'Meer,

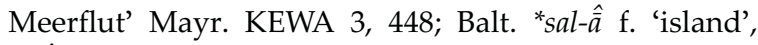
*sal- 'to flow' (Lith. salà 'Insel', dial. sálti 'fliessen'; Lett. sala 'Insel, Holm, eine Höhe im Morast'; OPrus. salus 'Regenbach' Mažiulis IV 55-56); Lat. insula f. 'Insel'. WP II 452, Pok. 899 (sub *sel- 'springen'). ? Here maybe as a loanword from an IE language (cf. Georgiev VS), Grk.

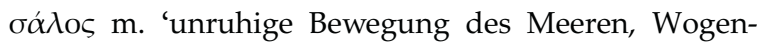
schwall; Ankerplatz, Reede'. WP II 454; Pok. 879-880, with irregular s-; nach Frisk II, 670, Beekes 1303-1304 vorgriechisches Word; Lat. salum (and salus Ennius) 'unruhiger Seegang, hohe See; Meer' WH 2, 471 (with Germ. as Wasserschwall etc.; but this is impossible, because ${ }^{*}$ swa- $>$ sua- in Latin! If the form of Ennius is primary, it may be a loanword from Greek, thus acc. to

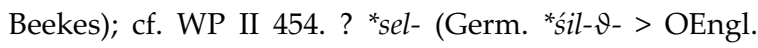
seolo» 'See'); \# Nostr. *calV 'wave': PA *čalu 'wave, to overflow' EDAL 391-392; Drav. *čal- 'well, spring' DED 2367.

PIH? PIE (Eur.)? * $a k^{w}$ - 'water (flowing)': Hitt. ? akukal(la?)- or akutal(la?)- 'Waschbecken' (Tischler 1112); Germ. *áxw-ō 'river, stream' f. Orel 5; Lat. aqua f. 'Wasser, Wasserleitung'. WP I 34 f, Buck 29, 35, 42. Pok 23, MA $636\left(h_{a} e k^{w} e h_{2^{-}}\right)$\# Nostr. *EEku 'water', PA *iák'a 'liquid, flow' or *uku 'wet, wash', Drav. SDr. *āk 'swamp' or *uk- 'spill, pour' МССНЯ 334, 347, ОСНЯ 1, 275-276. [two roots]

\section{River}

$\mathrm{PIH}{ }^{*} H a p$ - 'water, river': Hitt. hap(a)- 'Fluß', Pal. hāpnas, Luw. hāpinni- (Tischler 159-160) (MA - to ${ }^{*} h_{2} e b(h)$ - 'river'); InIr. *ap-/*āp- f. 'water, river' ЭСИЯ 1 ,

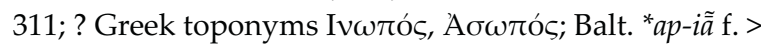
OPrus. ape 'Fluss', ap-us 'Brunnen' Mažiulis 1, 86-87, 89-90; Lat. amnis, -is m./f. 'Fluss, Strom, (dicht.) Strömung, Wasser'; Tokh. A, B āp 'water, river' (Adams 44). WP I 46, Buck 30, Pok. 51-52, MA 636 ( $h_{2} \bar{e} p-/ h_{2} e p$-) \# Nostr. * $V p V$ 'water', Ur *üptV (Redei 83) 'половодье', Drav NDr *op- 'become wet'. Or ? PIH *hab(h)- 'river': ? Hitt. hap (a)- 'Fluß' etc.; Celt. *ab-on Matasović 24-25 $\left(<\mathrm{PIE}{ }^{*} h_{2} e p-h_{3} \mathrm{on}\right.$, so related to $\left.\mathrm{PIH}{ }^{*} H a p-\right)$, Lat. amnis 'river'. Pok. 1, MA 486, Buck 1.36.

? PIE *dānu- 'river': IIr *dānu- (Ind. dấnu- n. 'fluid, drop, dew', Av dānu- f. 'Fluss, Strom'; Osset. don 'Wasser, Fluss') ЭСИЯ 3, 450-451; Wels donwy 'a river name' ( ${ }^{*}$ dānew-yos), Lat < Celt Dānuvius 'a river name'. Pok. 175, MA 486; WP I 763 (<*dā- 'to flow').

\section{Spring, well}

$\mathrm{PIH}^{*} g^{w}$ ela- 'to boil over': Hitt. kweluwana- (kuluwana-) 'Waschbecken' (Tischler 604); IIr. *gal-/*jal- 'to drip,
Altaic

PA (West) *orusi 'river, to flow': PT *örs, *örsen 'river'; PM *urus- 'to flow'. \# MCCHЯ 341: PIE Heur- / Hwer-, PK jwar-.

\section{River}

PA *āmu 'big river, big basin': ? PCT *umar 'big river'; PNM *ama- $n$ 'fold, valley'; PTM *āmu- 'lake; big river'; PKor *omi 'land sink, pool'; PJa *ùmí 'sea'. \# Cf. Dr. *am (Tamil am, àm) 'water' (DED 187).

PA (East) *kéba 'river, bay': PTung *xebe 'bay; lake'; PKor *kái ( < *kabi) 'inlet, estuary'; PJa *kápà 'river'.

PA *iuger'V 'river': PCT *ügür̆ 'small river'; PM *üjer 'flood, inundation'; PTM *uwgēe(r)- 'wave, stream'; PKor *jàhír 'shallow place'; PJa *ùrà 'bay, coast'. \# ? PU *uwa МССНЯ 334.

PA (West) *̌̌iōlu 'river bed, stream': PT *jul 'stream, brook, fountain'; PM *̌̌ilga 'river bed, ravine'; PTM *̌̌ila- 'swift (not freezing) river current, ice-hole'.

\section{Spring, well}

PA *biujri 'well, spring': PNM *bürü-dü 'spring'; PTM *bira 'river'; PKor “ù- 'well'; PJa *bì 'well'. 


\begin{tabular}{|c|c|}
\hline Indo-European & Altaic \\
\hline
\end{tabular}

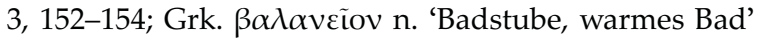
Beekes 195 ("Pre-Greek"); Germ. *kwellan- 'to gush, to drop' Orel 227. WP I 690, Pok. 471-472 \# Nostr *kütä 'pond', PA *kó́li, Ur. *k[ä]tV, Drav. *kUl- МCСНЯ 352, ОСНЯ 1, 305-306.

PIE *bhrew-eHr/*bhru-Hn-os (Gen.) 'spring (of water)': Arm. ałbiur, ałbeur, gen. ałber 'Quelle' Martirosian 32-33

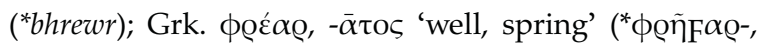

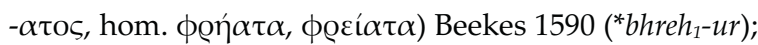
Germ. *brunn-ō(n-) f. 'spring, well' Orel 58. WP II 167, Buck 44, Pok. 144, MA 539 \# Nostr., PA *biujri 'well, spring'.

? PIE *alm-os 'spring': OInd. arma- 'Brunnen' Mayr. EWA 1, 120, ? Toch. B älme 'spring (of water)', vroddhi Toch B yolme 'Teich' (Adams 55-56). MA 539 ( ${ }^{*} h_{a}$ elmos, $\left.{ }^{*} h_{a} \bar{e} l m o s\right)$.

PIE *k(')rosn- 'stream, spring': Grk. aeol. к@á $v v \alpha$,

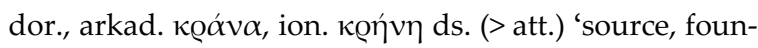
tain'; א@ouvós m. 'spring' Beekes 777; Germ. *xraznố 'tide, wave' Orel 185; Alb. krúa 'spring, fountain' Orel AlbD 198 (<*krāna < *krasna). WP I 488, Buck 44. MA 539 ( ${ }^{*} k_{o} s n e h_{a}{ }^{*}{ }^{*} k r o s n o-/ e h_{a}$, only Greek - Germanic) \# Nostr. *kara 'flood, spring', PA *k'ara.

PIE (Eur.) *bhog- 'brook, stream': Slav. *bāg-n-o 'marsh', ‘ $B \bar{a} g \bar{y}$ 'name of a river' Фасмер I, 102, Derksen 33 (not taking into account the Winter's law effect); Germ. *bak-iz m. 'stream' Orel 33; Celt. *boglā > MIr būal 'fliessendes Wasser'. WP II 187, Pok. 161 (the long vowel in Slav. is due to the Winter's law).

\section{To move across}

PIE *terH-, *trā- 'to move across': [Hitt. tarhu- 'besiegen, bezwingen, überwinden' is not here according Kloekhorst 835-837]; Ind. tárati, tiráti, titarti 'to pass across, cross over'; tára- 'carrying across or beyond', $\mathrm{m}$. 'crossing, passage'; taráni- 'moving forwards, carrying over'; táras- n. 'energy, progress; ferry'; tìrthá- n. 'Furt, Tränke' ( $\left.{ }^{*} t \bar{o} t h o-\right) ;{ }^{*} t \bar{u} r t h a ́-i n$ Prākr. tūha- 'Ufer', Dard. tūrt 'Furt' (> Wakhi tort Стеблин 368); Iran. Avest. tar- 'hinübererlangen über', prs. titar-, taraya-, ptc. vī-tarata-; tarantá- m. 'Meer'; OPers viyatārayāma 'wir überschritten'; ModPers. gu-dar 'ford', Bal. tarag, tharay 'umwen-

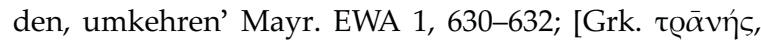

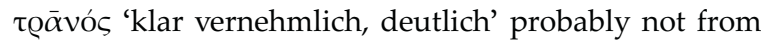
here, Beekes 1499]; Lat. intrāre 'hineingehen', extrō, -āre 'über etwas hinausgehen'. WP I 728, Pok. 1074-1075.

PIE (Eur.) * $b(h)$ red $(h)$ - 'to wade, to jump over': Slav. *brestī 'to wade', *brodz 'ford'; Balt. *brid- (breñd-a-) 'to wade', *brad-s-l-a- 'ford'; Alb. *breda 'to jump, to spring' Orel AlbD 34. Fraenkel 58, Schr-N I 167 \#
PA *biùlò 'to soak, to gush forth': PCT *bulak 'spring, well'; PNM *bilka- 'to issue from the ground, to overflow'; PTM *bilkü- 'to splash, swash'; PKor *puri- 'to soak, make wet'; PJa *pùrà 'bath'. \# PIE *bhleu- (WP 2, 213-214).

\section{To move across}

PA *tiùge 'ford, bridge': PNM *tuguj 'brow, gangway'; PTung *tügde- 'to cross a bridge; sb. bridge, log'; PJa *tù 'ford'.

PA (East) *t'olV 'bridge, crossing': PTung *tul- 'to wade; to cross (a mountain ridge)'; PKor *tằri 'bridge'.

PA *ól'a 'ford, shallow': PNM *(h)olam 'ford'; PTM *ola- 'to ford, wade'; PJa *ásá- 'shallow'.

PA *kop ira 'bridge, crossing': PCT *köpür 'bridge'; PM *köjürge 'bridge'; PTM *xupuru 'rift (in river); bridge'; PJa *kápárá 'shallow, sandy place in a river or on its bank'.

PA (East) *bét a / *péda 'sea, ford': PTM *pede-- 'to ford, cross over'; PKor *pàtá, *pàrắr 'sea'; PJa *bátá 'sea; to ford'. 


\begin{tabular}{l}
\hline \multicolumn{1}{c|}{ Indo-European } \\
\hline Nostr. *bVrdV 'ford', PA *bét'al*p'éda, Kart. *bo(r)d-, Drav. \\
*pad- MCСНЯ 332. \\
? PIE *telat- 'bridge, ford': ? Ind. tīrthá- n. 'ford' (if \\
not from *terH- 'to move across'); Balt. *tilt-a-s 'bridge'. \\
Fraenkel 1094 (differently in Pok.), Schr-N I 167, \\
Иллич-Свитыч 1963, 74.
\end{tabular}

PIE (Eur.) *bher[e]w-, *brēw- 'wooden flooring, decking, bridge': Slav. *brbvb-no 'beam, bridge' Derksen 67; Germ. *brốw-ō, *bru-g-jōn- 'bridge' Orel 58; Celt. *brēwā > Gaul. briva 'Brücke'. WP II 207, Pok. , Schr-N I 167. \# Nostr.: Ur. *pora 'raft, float' UEW 395. МССНЯ 332.

PIE *pont-/*pent-/*pnt- 'way through obstacles, way through water space': Ind. pánthā 'way, path'; päthas- n., pāthis- n. 'spot, place; (L.) water'; Avest. pant̄̄, instr.

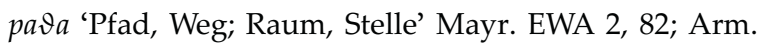
*fon-i- > hun (i-St.) 'Furt, Weg' Martirosian 422-426; Grk.

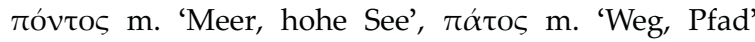
Beekes 1221; Slav. *pōts 'way' Derksen 417, Balt. *pint-[i]> OPrus. pintis 'way, road' Mažiulis 3, 281-282, Иллич-

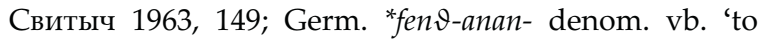
find' Orel 99; Lat. pons, -tis m. 'Brücke, Steg, Prügelweg durch Sümpfe, Verdeck, Schiffstabulat' WH II 336; ? Celt. *fanssā 'trace' > OIr. ès 'Spur' Matasović 121 (< *pnt-teh ${ }_{2}$ ); ? Tokh. B -pänte as a putative PIE *pnth ${ }_{2}-o$ - 'one pertaining to the way' Adams 19. WP II 26; Pok. 808-809; Benveniste 1954.

\section{Still water}

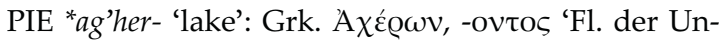

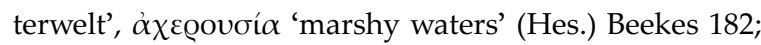
? Arm. ezr, Pl. *ezer-a (*n.) 'edge, bank' Martirosian 247249; Slav. *e/ozero, *ezerb 'lake' Derksen 148, Trub. 6, 57, 59 (but the suggested connection with Slav. *ězr 'dam, fishing basket' is doubtful because of different vowel quantity); Balt. *ežer-an 'lake' n. Fraenkel 125, Mažiulis 1, 104 (but the suggested connection with Balt. * $e \check{z}-j \hat{\bar{a}},-i \tilde{\bar{a}}$ f. 'border' is not obligatory). Pok. 291-292; MA 343 ( ${ }^{*} h_{1}$ eg'herom without Greek and Arm.).

? PIH *woHp-: *wōp-/*up 'basin': Hitt. wappu 'river bank' Kloekhorst 958 ("no good etymology”); OInd. vāpí 'pond' (Mayr. KEWA III 188: to váp- 'to throw, to sow'); OCSl vapa 'lake, marsh, pond' Фасм. 1, 125; ? Lith. ùpe, Lett. upe 'river' (the short $u$ is unclair). Pok. 1149, MA 343, 636-637 (uncertain).

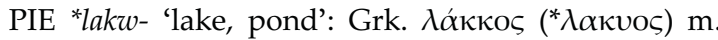
'Wasserloch, Zisterne; Teich, Grube' Beekes 827 ( $\left.{ }^{*} l k w-\right)$; Slav. *loky 'pool, swamp, pond' Derksen 284; Balt. *lek-

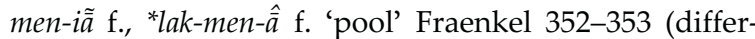
ently); Germ. *lagú-z 'sea, lake’ Orel 231; *láx-ō, *láx-az 'sea, pool, swamp' Orel 232; Lat. lacus, gen. - $\bar{u} s(/-\bar{\imath}) \mathrm{m}$. 'jede trogartige Vertiefung, See; Brunnentrog; Kufe;
Altaic

\section{Shallow place}

PA *niala 'shallow, shallow place': PCT *jAl- 'shallow; wave'; PNM *naliyur 'pool; overflowed plain'; PTung *niala 'overflowed place; shallow'; PKor *nằră 'ford; ferry point'.

PA *siógu 'shallow, shallow place': PCT *sig 'shallow'; PM *siga-r 'sediments'; PTung *sigi-n 'ice-hole'; PJa *sú 'shallow place, sandbank'.

PA *sájV 'pebble; shallow place': PT *saj ' shallow place with pebbles; arroyo with pebbles; wadi; river'; PNM *sajir 'river-bed, pebble'; PTung *saj- 'sandy mound'; Kor. *sŭai-m 'spring, shallow well'; PJa. *sái 'sandbank'.

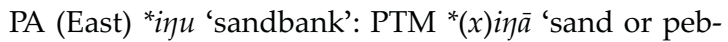
ble on the riverbank, sandbank; spit'; Kor. *jā 'reef, rock in a sea'; PJa. *ía 'bay'.

\section{Still water}

PA *kóoll 'lake, basin': PT *[k]ōl 'lake'; PNM *küjil-sü 'island in a river, shallow place in a river'; PTM *xule'canal, duct; whirlpool'; PKor *kằrắm 'lake, big river'. \# ? PIE * $g^{w} \mathrm{el}$ - 'quellen, Quelle' (WP 1, 690); PU * $k[\ddot{a}] t V$; Drav *kul a (МССНЯ 352, ОСНЯ 1, 305-306).

PA *najrV 'lake, river': PM *narur 'lake'; PTM *niāru 'lake; swamp'; PKor *nāih 'river'. \# ОССНЯ 2, 89.

? PA (West) *žàdé ( *žèdá) 'pond, pool': PNM *žadarai 'pond, pool'; ? PJa dàntá 'backwater'. 


\begin{tabular}{c|c}
\hline Indo-European & Altaic \\
\hline
\end{tabular}

Grube'; lacūna f. 'Vertiefung, Senkung; Loch, Grube; Lache, Weiher'; Celt. *loku 'lake, pool' Matasović 243. WP II 380, Pok. 653, MA 343 (without Balt.). \# Nostr.: Ur. *lake ' bay, low ground' UEW 234, 683.

PIE (Eur.) *lām- 'hollow filled with water': Slav. *lāms 'pit, flooded meadow' ЭССЯ 14, 26, Derksen 268 (to *lomiti 'to break'); Balt. *lâa $m$ - $\hat{\bar{a}}$ (1?), *lām-a- 'hole, den, pit' Fraenkel 385 (to laminti etc.; doubtful because of different vowel quantity); Lat. lāma f. 'Lache, Morast, Sumpf' WH I 753. WP II 385, Pok. 653-654. \# Nostr. *laHm[u] 'swamp', PA *làmò 'sea, wave', Ur. *lampe 'pond', Kart. *lam- 'silt, dampness', Drav. *nam 'dampness'. МССНЯ 331, ОСНЯ 2, 29-30.

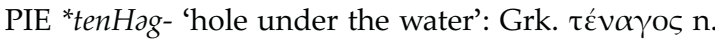
'shallow place' Beekes 1466; Balt. *ting- $\hat{\bar{a}}>$ Lett. tīgas pl. 'Tiefe zwischen zwei Untiefen'. WP I 724, Pok. 1067, MA 343 (tenh ag- $_{\text {t }} n h_{a} g-$ ).

PIE (Eur.) *lindh- 'reservoir': Germ. *lindō 'spring, pool, wave' Falk \& Torp 244, De Vries 357; Celt. *linda 'lake' Matasović 239-240. WP II 438, Pok. 675.

\section{Sea}

PIE * $m o H r-{ }^{*} m \bar{r}-{ }^{*} m_{\partial} r$ - 'sea, lake'10: Ind. mìra- m. 'the sea, ocean; (L. also) limit, boundary’ Mayr. KEWA 2, 644; Iran. Ossetic mal < *māri-, *māryo- 'deep still water, deep place in a bassin; fig. a giant quantity of fluid' Аб. II 68-69; Slav. *morje 'sea' Derksen 325; Balt. *mar-i,

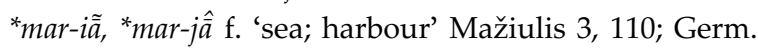
*mariz, *marīn 'sea, lake', *marisk-a-z m. 'marsh' Orel 261, *mōr-a-z m., n., *mōr-i-z 'marsh, lake, sea' Orel 274; Lat. mare, -is n. 'Meer'; Celt. *mori 'sea' Matasović 277. WP II 234, Pok. 748, MA 2, 503 ( ${ }^{*}$ mori; + Arm. mawr < ? *maru 'marsh', but see Martirosian 447 - not here). \# Nostr. *märä 'wet', PA mì̀ ri 'water', Kart. *mar-, Drav. *mar- МССНЯ 334, ОСНЯ 2, 60-61.

? PIE *g'wop- ( -bh-) 'sea': Arm. cov 'Meer' Martirosian 141; Germ. *kwa[f]-a- > ON kaf, OSwed. kwaf 'Meerestife' n. De Vries 296 (=kaf 'untertauschen'). WP I 637, Pok. 465-466.

[PIH *g'rei- 'to spread' > 'big water surface': Hitt. karaitt-/karett- c. 'flood, inundation' Kloekhorst 440 ( ${ }^{*}$ 'roi-t- / *g'rei-t-); Ind. jráyati 'stürmt an, läuft an', jráyas- n. 'Ungestüm, Lauf, Flußlauf'; Av. zrayah-, OPers. drayah- 'See, Meer', MPers. zray, ModPers. daryā 'sea, big river' Mayr. EWA 1, 606. WP I 660, Pok. 401 ("Nur

\section{To overflow, deep water}

PA *dấla 'deep place': PT *dāl- 'to sink' > *dAluj 'ocean'; PTM *dala(n) 'inundation, stream'; PJa *dara 'sea bottom, deep place'.

PA *t'òle 'deep water': PCT *tolku- 'to beat (of waves); wave(s)'; PNM *tülki-n 'rising water'; PNTung *tōlgu'deep place close to the bank; whirlpool; backwater'; PKor *tór 'ditch'; PJa *tara 'backwater, deep water'.

PA *kara 'to overflow, flood': PCT *KAr- 'to overflow'; *KArïm 'ditch'; PNM *kargi- 'overfall, rift'; PTung *xarba 'shallow place, shoal; shallow; ebb-tide'; PJa *kátà 'tide, ebb-tide; beach, bay'.

PA (West) *tiál'ke 'to splash, overflow': PCT ${ }^{*} d(i) \bar{a} \lambda(i)$ - 'to overflow'; PNM *čalgi- 'to splash, overflow'; PTung *tilka- 'to splatter, overflow'.

PA *ōke 'deep place, place far from the shore': PT *ōkü 'hole in ice'; PNTung * $(x) u K$ - $t$ - 'ice-hole; river rift'; PJa * $k i$ 'open sea'.

PA *làmo 'basin, wave': PNM *namug 'marsh, swamp'; PTM *lāmu 'sea; wave'; PJa *nàmì 'wave' \# PIE *lām-, PU *Lampe, PD *namV, Georg. lam- (МССНЯ 331, ОСНЯ 2, 29-30).

${ }^{10}$ Cf. Гамкрелидзе \& Иванов 1984 673; the Hittite form marmara-, mammara- only means 'a type of landscape' (Friedrich 137, Tischler 3, 140-141), so it is not necessarily a reflexation of this root; if so, the more probable proto-meaning is 'sea': European - Indo-Iranian, cf. Sanskrit mīra-, mirāa- 'sea; boundary' (cf. Mayr. II 644), Osset. *mal < *māri-, despite Гамкрелидзе \& Иванов 1984673 not 'stagnant water', rather 'deep place in a bassin; a giant quantity of fluid', cf. mal of blood etc., see Аб. II 68-69. 


\begin{tabular}{|c|c|}
\hline Indo-European & Altaic \\
\hline 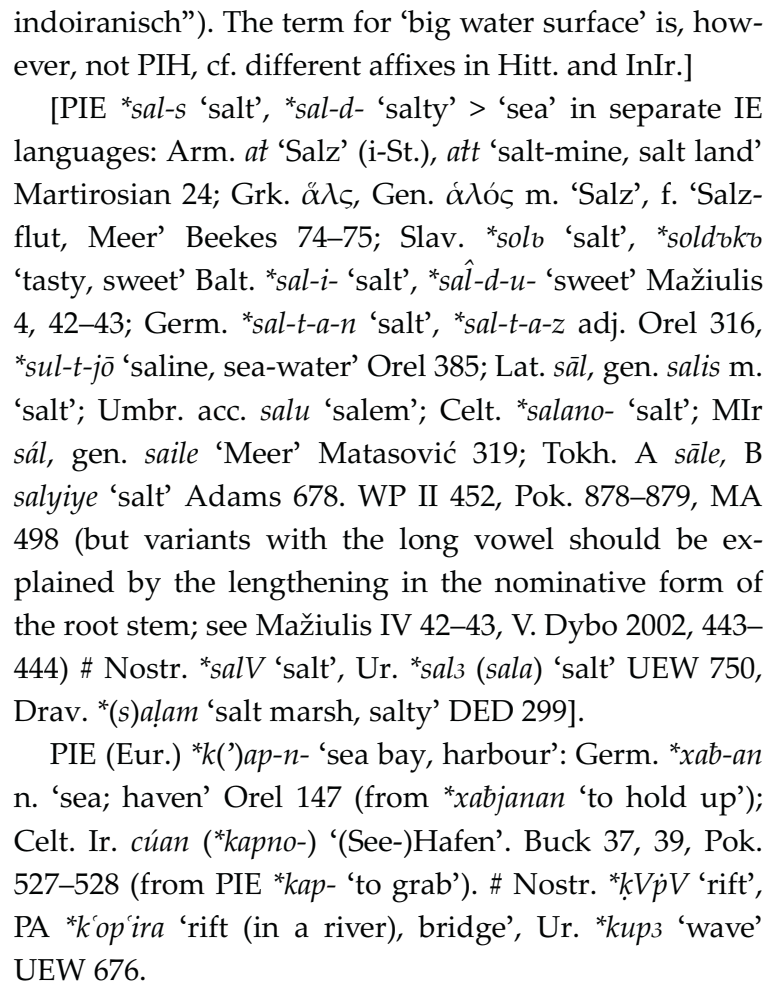 & $\begin{array}{l}\text { Salt } \\
{[\text { PA *čiober'V 'salt; bitter, acid': PT *dū ř 'salt'; PM }} \\
\text { *dabusu 'salt'; PTM *̌̌ujar- 'bitter, acid'; PKor *čjōr- } \\
\text { 'salty'; PJa *túrá- 'hard, bitter'. \# Dr. *suvar 'salt, brack- } \\
\text { ishness; salty' (DED 2674)]. }\end{array}$ \\
\hline $\begin{array}{l}\text { Foam } \\
\text { PIE *(s)poyHmn- (or rather *(s)poHymn-, cf. Balt.; see } \\
\text { in detail V. Dybo 2002, 389) 'foam': Ind. phéna- m. } \\
\text { 'foam' Mayr. EWA 2, 204; Ir. *faina-ka 'foam' ЭСИЯ III } \\
\text { 44; Slav. *pěna A ‘foam' Derksen 397; Balt. *spáin-ia (1) > } \\
\text { *spaĩn-ia (Hirt's law) 'foam' Fraenkel } 1 \text { 858, Mažiulis IV } \\
\text { 128; Germ. ‘faim-an n. 'foam' Orel 90; Lat. spūma f. } \\
\text { 'Schaum, Gischt' ( }{ }^{*} \text { spoima) WH II, 580. WP II 681, Pok. } \\
\text { 1001. }\end{array}$ & $\begin{array}{l}\text { Foam } \\
\text { [PA *kóp 'i 'to foam': PT *köp- 'to foam, to swell'; PM } \\
\text { *köjye- 'to foam, swell up'; PTM *xapu- *xopu- 'foam'; } \\
\text { PKor *kàphúm 'foam']. } \\
\text { [PA *ùjbà 'foam': PM *ibil- 'to flow (of milk from the } \\
\text { udder at the time of sucking)'; PTM *(x)ōb- 'to get cov- } \\
\text { ered by foam; foam (on water)'; PJa *àwà 'foam']. }\end{array}$ \\
\hline
\end{tabular}

Based on these juxtapositions for a number of proto-lexical microsystems, the following conclusions can be proposed.

The peculiarities of the landscape-related lexicon in both families are as follows. First of all, the steppe must be excluded from the regions potentially inhabited by Proto-IndoEuropeans. ${ }^{11}$ Some relatively high mountains with many kinds of rocks and sharp or big stones are present. ${ }^{12}$ Some of these mountains are covered by forests. There are words for narrow passages, canyons, precipices, mines and caves, foothills, valleys and dells, meadows in

${ }^{11}$ When I say - more or less arbitrarily - about the "Proto-Altaics" and "Proto-Indo-Europeans", each of these labels surmises a big human community whose members are territorially and culturally related: hypothetic ethnos-speakers of the Proto-Indo-European language, reconstructed for the initial period of its disintegration into separate groups of languages, and, likewise, hypothetic ethnos-speakers of the Proto-Altaic language, reconstructed for the same period. The contrastive list of Proto-Indo-European and Proto-Altaic thesauri in selected thematic areas is still preliminary; conclusive results will be obtained upon systematic application of semantic reconstruction to all the subgroups of related languages.

${ }^{12} \mathrm{Cf}$. the identical conclusion, reached on different grounds by Гамкрелидзе \& Иванов 1984. 
forests and on the river-banks. The rivers have fords and are definitely smaller than their Proto-Altaic counterparts (there is no semantic variation between "river" and "sea"; nota bene that the only trace of the name of flood is GA; the lower Danube?); cf. here the noticeably weaker function of fish in the Indo-European economy (expressed in a substantially smaller number of terms for fishing tools, fish body parts and fish species - see the example below). But they could have lived near a sea or a big lake with sandy banks ${ }^{13}$.

In Proto-Altaic, the landscape was represented by names of not very high mountains, lowpitched slopes, foot-hills (also with rocks and gravel). They had a number of verbs meaning "to cross mountains." The canyons, valleys and steppes are present, the steppes being of a rather arid and dusty type. There are many terms for small, quick-flowing rivers with shallows and rifts, but we also know words for big rivers as well. The reflexes of those words reveal semantic variation: in some daughter languages they mean "big river", while in the others the meaning is "sea". We may talk about islands and floods. Floods, from my point of view, may rather indicate big rivers with seasonal floods. We still do not have a reliable reconstruction with the undisputable meaning of "sea". The Tungus-Manchu name for sea can be traced back to a common name for wave, while the common Korean and Japanese forms originally meant "ford". The same development is found, for example, in Ancient Greek, where the corresponding word is historically explained as a development of the original meaning "aquatic way".

\section{Fishing tools}

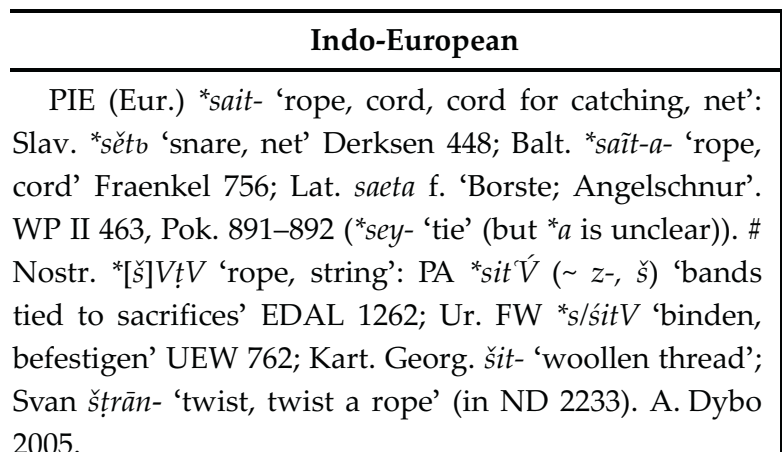

?? PIH*h $h_{1}$ ekt- 'net': Hitt. ekt- 'hunting net', Luv. aggati- 'catch-net' Kloekhorst 235-236 (to OHG jagōn 'to hunt', PIE *jek-); Ind. ákṣu- 'net' Mayr. EWA 1, 42 (to ákṣi

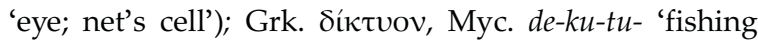
net' Beekes 335-336 (Pre-Greek). MA 2, 393 (“ $d$ - as in 'tear'”).

? PIE (GA) *pork'- 'fish-net, noose': Arm. ors 'hunt, catch' Martirosian 544 (to *york-os 'roe'); Grk. тó@коs m. 'Art Fischernetz' Beekes 1222. WP II 44 \# Cf. PA *p ürVk $V$ 'rope, lasso'.

PIE (Eur.) *wadh- 'fish net': Slav. *vodt; *nevodt 'dragnet' Фасм. 3, 55-56; Balt. *wad-a-, -u- . 'grosses Zugnetz; Flügel eines Zugnetzes’ Fraenkel 1177; Germ.

\section{Altaic}

PA *tóbru 'catching net': CT *Tuřak 'trap'; PM *towr 'net, cage'; PTung *turku- 'to get caught (in a trap, net)'; PKor *tằràchí 'basket'; PJa *túrí 'fishing'.

PA*àgya 'net': PCT *āg 'net'; PNM *aro-ga 'leading string in net'; PTung *ayga 'net (for catching fish under ice)'; PJa *àmì 'net', OJa ama 'fisherman', cf. *àm- 'to knit'.

PA (West) *nable 'net, fish-trap': PT *jilizm 'fishing net'; PTung *nalba 'fish-trap, crib'.

PA (West) *iuji 'to weave (nets), net': PNM *(h)öje-si 'fishing net'; PTung *inji- 'to weave net'.

PA (West) *t'ukV 'fishing dam, fishing net': PT *Tug 'dam, fish-trap'; PNM *togsija 'bird net, fish net'; PSTung *tuki- 'to fish by a stake net in a narrow channel'.

PA *gòlí 'a k. of tool for water-hunting': PM *gol-mi 'net'; PTung *goli 'net for big fishes'; PJa *kùrúrí 'arrow for shooting sea-birds or for catching fish'.

PA *bé 'bait': PCT *be- $\eta$ 'bait, bird-seed'; PTM *be 'bait'; PJa *bái 'bait'.

PA *ill'bi 'fish bait': PNM *(h)ilbeye-sün 'fish bait'; PTM *īlbī 'bait; plummet, sinker'; PJa * $i(n) s a-r$ - 'to fish'.

${ }^{13}$ Cf. Гамкрелидзе \& Иванов 1984: 673-674; they are definitely right that the IE names for 'sea' could originally be the names for some big and deep lake, but the existence of names for 'salt basins' derived from the IE name for 'salt' in a number of IE languages is not necessarily proof of the fact that Proto-Indo-Europeans must have known salt lakes or seas. Cf. the similar conclusion in MA 498. 


\begin{tabular}{|c|c|}
\hline Indo-European & Altaic \\
\hline 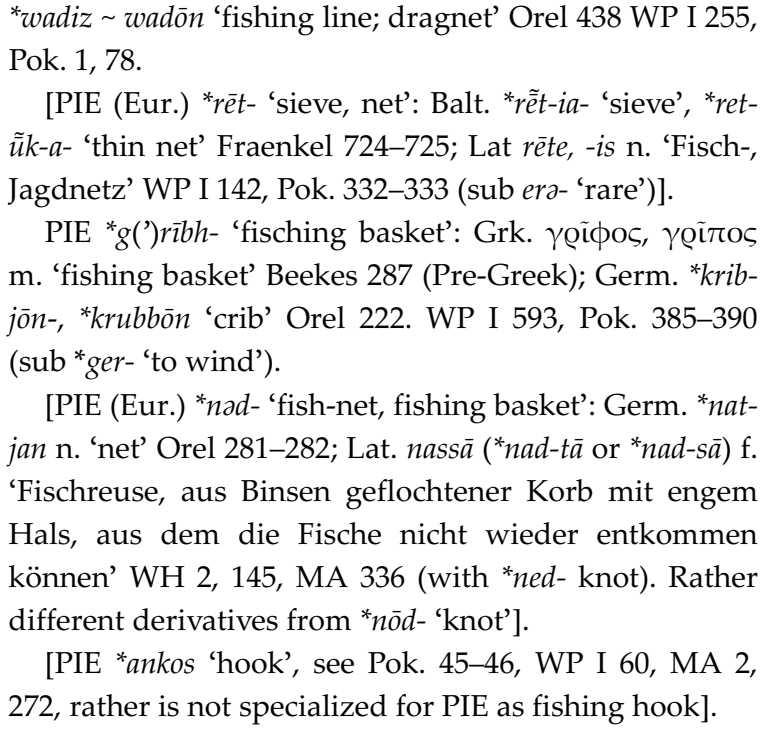 & \\
\hline $\begin{array}{l}\text { On the whole } 1 \text { reliable PIE term for fishing net, } 1 \text { re- } \\
\text { liable Eur. term for fishing net, } 1 \text { reliable Eur. term for } \\
\text { fishing basket, } 1 \text { reliable Eur. term for a fishing tool. }\end{array}$ & $\begin{array}{l}\text { On the whole } 2 \text { reliable PA terms for fishing net, } 2 \text { re- } \\
\text { liable PA terms for bait, one reliable PA term for a fish- } \\
\text { ing tool; } 3 \text { reliable Western Altaic terms for fish traps. }\end{array}$ \\
\hline
\end{tabular}

We can also see that a substantial part of landscape environment terms can be reconstructed only for the later stages of PIE. The set of terms that is reconstructed for PIH is hardly telling: PIH *dg'hom/*dg'hem 'earth, soil, territory, earth surface'; PIH *wedn-/*udn- 'earth, soil, territory'; PIH *pē(n)s- 'sand, pebble'; PIH ${ }^{*} h_{2} e k(') h_{2}-m o n,{ }^{*} k \bar{a}-m o n$ (< $\left.{ }^{*} k e h_{2}-m o n-\right)$ 'stone, rock'; $\mathrm{PIH}{ }^{*} H a r$ - 'valley, vale, dale; grotto; swamp'; PIH *peru-(n-) 'mountain top'; PIH *kolHn-, *kolHm- 'top, hill, rock'; PIH *weHr-/uHr- 'water, moisture'; PIH *we(n)dh- 'water, wave'; PIH *Hap- 'water, river'; $\mathrm{PIH}^{*} g^{w}$ ela- 'to boil over'; ? $\mathrm{PIH}$ *woHp- 'basin'. Nonetheless, even in this case we see mountainous terrain entering the picture.

\begin{tabular}{lll} 
& \multicolumn{1}{c}{ Abbreviation s } \\
Alb - Albanian & Grk. - Ancient Greek & \\
Arm. - Armenian & Ark. - Arkadian & MIr - Middle Irish \\
Av - Avestan & Att. - Attic & MPers. - Middle Persian \\
Bal. - Baluchi & Corcyr. - Corcyrean & Nostr - Nostratic \\
Balt. - Baltic & Dor. - Doric & NPers. - New Persian \\
Bret. - Breton & Ion. - Ionic & ODalm. - Old Dalmatian \\
Celt-Ital - Celto-Italic & Lesb. - Lesbian & OInd. - Old Indian \\
Corn. - Cornish & Hitt. - Hittite & OIr - Old Irish \\
Cymr. - Cymrish & Ind. - Indian & OLith. - Old Lithuanian \\
Dard. - Dardic & Iran. - Iranian & OPrus - Old Prussian \\
Drav - Dravidian & Kart - Kartvelian & Osset. - Ossetic \\
ESlav. - East Slavic & Kor. - Korean & OSwed - Old Swedian \\
Eur. - European & Kur. - Kursh & PA - Proto-Altaic \\
GA - Greek-Aryan & Lat - Latin & Pal. - Palaic \\
Gael. - Gaelic & Lett. - Lettish & PCT - Proto-Common Turkic \\
Georg - Georgian & Lith. - Lithuanian & PIE - Proto-Indo-European \\
Germ. - Germanian & Luw. - Luwian & PIH - Proto-Indo-Hittite \\
& & PIIr - Proto-Indo-Iranian
\end{tabular}




$\begin{array}{ll}\text { PIran - Proto-Iranian } & \text { PT - Proto-Turkic } \\ \text { PJa. - Proto-Japanese } & \text { PTM - Proto-Tungus-Manchu } \\ \text { PM - Proto-Mongolian } & \text { PTung - Proto-Tungussic } \\ \text { PNM - Proto-North Mongolian } & \text { Rum. - Rumanian } \\ \text { PNTung - Proto-North Tungussic } & \text { Slav. - Slavic } \\ \text { Prakr. - Prakrit } & \text { Tokh - Tokharian } \\ \text { PSTung - Proto-South Tungussic } & \text { Ur.- Uralic }\end{array}$

VLat. - Vulgar Latin

\# - supposed external relation

$[\ldots]-$ The reconstructed stem does not belong to the considered semantic area

\section{Literature}

Adams - AdAms D. Q. A dictionary of Tocharian. Amsterdam - Atlanta, 1999.

Beekes - BEEKES R. Etymological dictionary of Greek. Leiden: Brill, 2010.

Benveniste 1954 - BENVENISTE É. Problèmes sémantiques de la reconstruction // Word 1954, Vol. 10, № 2-3.

Benveniste 1970 - BENVENISTE É. Le vocabulaire des institutions indo-européennes. I: Économie, parenté, société. II: Pouvoir, droit, religion. Paris, 1970.

Buck - BUCK C. D. A dictionary of selected synonyms in the principal Indo-European languages. Chicago, 1949.

Blažek 1992 - BLAŽEK V. Kartvelian Material in Nostratic Lexicon: New Etymologies. Nostratic, Sino-Caucasian, Austric and Amerind. Bochum: Brockmayer, 1992. [I] 112-128, [II] 129-148.

De Vries - DE VRIES J. Altnordisches etymologisches Wörterbuch. Leiden, 1957-1961.

DED - BurRow Th., EMENEAu M. A Dravidian etymological dictionary. $2^{\text {nd }}$ ed. Oxford, 1984.

Derksen - DERKSEN R. Etymological dictionary of the Slavic inherited lexicon. Leiden: Brill, 2008.

A. Dybo 2005 - Dyво A. V. Some peculiarities of Altaic reflexes of Nostratic sibilants // Nostratic Centennial Conference. The Pecz Papers. Pecz, Lingua franca Group, 2005. P. 85-114.

V. Dybo 2002 - Dyвo V. A. Balto-Slavic Accentology and Winter's Law // Studia Linguarum 3/2. Moscow, 2002.

EDAL - Starostin S. A., DYBo A. V., MudraK O. A. The etymological dictionary of Altaic languages. Leiden: Brill, 2003.

Falk \& Torp - Wörterbuch der Indogermanischen Sprachen: Dritter Teil: Wortschatz der Germanischen Spracheinheit by August Fick with contributions by Hjalmar Falk, entirely revised by Alf Torp in 1909.

Fraenkel - FRAENKEL E. Litauisches etymologisches Wörterbuch. Bd. I-II. Heidelberg, 1965.

Friedrich - FRIEDRICH J. Hethitisches Wörterbuch. Heidelberg, 1952-1954.

Friedrich 1979 - FRIEDRICH P. Indo-European names of trees. Chicago 1979.

Frisk - FRISK H. J. Griechisches etymologisches Wörterbuch. Heidelberg, 1954.

Georgiev VS - GEORGIEV V1. Vorgriechische Sprachwissenschaft. Vol. 1-2. Sofia, 1941-1945.

Holthausen - HolthAUSEN F. Altenglisches etymologisches Wörterbuch. Heidelberg, 1934.

Kloekhorst - KLOEKHORST A. Etymological Dictionary of the Hittite Inherited Lexicon. Leiden-Boston: Brill, 2008.

MA - AdAms D. Q., MALlory J. P. (ed.). Encyclopedia of Indo-European culture. Chicago, 1997.

Mallory \& Adams 2006 - Mallory J. P. \& Adams D. Q. The Oxford Introduction to Proto-Indo-European and the Proto-Indo-European World. Oxford: Oxford University Press, 2006.

Martirosian - MartiRosian G. Etymological dictionary of the Armenian inherited lexicon. Leiden: Brill, 2010.

Matasović - R. MATASOvić. Etymological Dictionary of Proto-Celtic. Leiden-Boston: Brill, 2009.

Mayr. EWA - MAYRHOFER M. Etymologisches Woerterbuch des Altindoarischen. Heidelberg, 1986-1996.

Mayr. KEWA - MAYRHOFER M. Kurzgefasstes etymologisches Wörterbuch des Altindischen. Heidelberg, $1953-1979$.

Mažiulis - MAŽıUlis V. Prūsu kalbos etimologijos žodinas. I-IV. Vilnius: Mokslas, 1988-1997.

Melchert CLL - MelChert H. Craig. Cuneiform Luvian lexicon. Chapel Hill, 1993.

ND - Dolgopolsky A. The Nostratic Etymological Dictionary. Preliminary electronic publication. McDonald Institute for Archaeological Research, 2008.

Orel - Orel V1. A Handbook of Germanic Etymology. Leiden/Boston: Brill, 2003.

Orel AlbD - Orel Vl. Albanian Etymological Dictionary. Leiden/Boston/Koln: Brill, 1998.

Pok. - POKORNY J. Indogermanisches etymologisches Wörterbuch. Bern, 1959.

Renfrew 1987 - RENFREW C. Archaeology and language. The puzzle of Indo-European origins. London, 1987.

Schrader \& Nehring - SCHRADER O., NEHRING A. Reallexikon der indogermanischen Altertumskunde. I-II. Berlin/Leipzig, 1917-1928. 
Sihler - SiHLER A. L. New comparative grammar of Greek and Latin. Oxford, 1995.

Tischler - TISCHLER J. Hethitisches etymologisches Glossar. Insbruck, 1977-.

Trautmann - TRAUTMANN R. Baltisch-Slawisches Wörterbuch. Göttingen 1923.

Turner CDIAL - TURNER R. L. A Comparative Dictionary of the Indo-Aryan Languages. London, 1962-1964.

UEW - REDEI K. Uralisches etymologisches Wörterbuch. Budapest, 1986-1989.

WH - Walde A., Hofmann J. B. Lateinisches etymologisches Wörterbuch. Bd. I-II. 5. Aufl. Heid., 1982.

WP - WALDE A. Vergleichendes Wörterbuch der indogermanischen Sprachen. Hrsg. u. bearb. von J. POKORNY. Bd. I-III. Berlin/Leipzig, 1926-1932.

Аб. - АБАев В. И. Историко-этимологический словарь осетинского языка. Т. I-V. Москва/Денинград, $1958-1995$. [ABAEV V. I. Istoriko-etimologicheskij slovar' osetinskogo yazyka. T. I-V. Moskva/Leningrad, 1958-1995.]

АЭ - Алтайские этимологии. Ленинград, 1984. [Altajskie etimologii. Leningrad, 1984.]

Гамкрелидзе \& Иванов 1984 - ГАМКРЕдИДЗЕ Т. В., ИвАНОВ Вяч. Вс. Индоевропейский язык и индоевропейцы. T. 1-2. Тбилиси, 1984. [GAMKRELIDZE T. V., IVANOV Vyach. Vs. Indoevropejskij yazyk i indoevropejcy. T. 1-2. Tbilisi, 1984.]

А. Дыбо 1996 - ДыБо А. В. Семантическая реконструкизия в алтайской этимологии. Москва, 1996. [DYво А. V. Semanticheskaya rekonstrukciya v altajskoj etimologii. Moskva, 1996.]

А. Дыбо 2000 - ДыБо А. В. Ностратические этимологии с начальными носовыми // Проблемы изучения дальнего родства языков на рубеже 3-го тысячелетия. Тезисы и доклады конференции. Москва: РГГУ, 2000. C. 31-38. [Dүво A. V. Nostraticheskie etimologii s nachal'nymi nosovymi // Problemy izucheniya dal'nego rodstva yazykov na rubezhe 3-go tysyacheletiya. Tezisy i doklady konferencii. Moskva: RGGU, 2000. S. 31-38.]

А. Дыбо 1997 - ДыБО А. В. К культурной лексике праалтайского языка // Балто-славянские исследования 1988-1996. Москва, 1997. [DҮво A. V. K kul'turnoj leksike praaltajskogo yazyka // Balto-slavyanskie issledovaniya 1988-1996. Moskva, 1997.]

Иллич-Свитыч 1963 - Илдич-Свитыч В. М. Именная акцентуация в балтийском и славянском. Москва, 1963. [ILliCH-SviTYCH V. M. Imennaya akcentuaciya v baltijskom i slavyanskom. Moskva, 1963.]

ИОЭАЯ - Исследования в области этимологии алтайских языков. Ленинград, 1979. [Issledovaniya v oblasti etimologii altajskikh yazykov. Leningrad, 1979.]

МССНЯ - Иллич-Свитыч В. М. Материалы к сравнительному словарю ностратических языков // Этимология 1965. Москва, 1967. [ILLICH-SviтYCH V. M. Materialy k sravnitel'nomu slovaryu nostraticheskikh yazykov // Etimologiya 1965. Moskva, 1967.]

Невская 1977 - НевскАЯ Л. Г. Балтийская географическая терминология. Москва: Наука, 1977. [NEVSKAYA L. G. Baltijskaya geograficheskaya terminologiya. Moskva: Nauka, 1977.]

ОСААЯ - Очерки сравнительной лексикологии алтайских языков. Ленинград, 1972. [Ocherki sravnitel'noj leksikologii altajskikh yazykov. Leningrad, 1972.]

ОСНЯ - Илдич-Свитыч В. М. Опыт сравнения ностратических языков. Т. І: Введение. Сравнительный словарь b-k. Москва, 1971; Т. ІІ: Сравнительный словарь l-n. М., 1976; Т. ІІІ: Сравнительный словарь p-q. Москва, 1984. [ILlich-Svitych V. M. Opyt sravneniya nostraticheskikh yazykov. T. I: Vvedenie. Sravnitel'nyj slovar' b-k. Moskva, 1971; T. II: Sravnitel'nyj slovar' l-n. M., 1976; T. III: Sravnitel'nyj slovar' $p-q$. Moskva, 1984.]

Старостин 2007 - Старостин С. А. Труды по языкознанию. Москва, 2007. [StArostin S. A. Trudy po yazykoznaniyu. Moskva, 2007.]

Стеблин - СТЕБАИН-КАМЕНСКИЙ И. М. Этимологический словарь ваханского языка. Санкт-Петербург, 1999. [STEBLIN-KAMENSKIJ I. M. Etimologicheskij slovar' vakhanskogo yazyka. Sankt-Peterburg, 1999.]

Толстой 1969 - ТолстОй Н. И. Славянская географическая терминология. Москва, 1969. [TOLSTOJ N. I. Slavyanskaya geograficheskaya terminologiya. Moskva, 1969.]

Топоров - Топоров В. Н. Прусский язык. 1-5. Москва, 1975-. [TOPORоv V. N. Prusskij yazyk. 1-. Moskva, 19751990.]

Фасмер - ФАсмеР М. Этимологический словарь русского языка. В 4-х томах. Москва, 1964-1973. [FASMеR M. Etimologicheskij slovar' russkogo yazyka. V 4-kh tomakh. Moskva, 1964-1973.]

ЭСИЯ - РАСТОРГУЕВА В. С., ЭДЕАЬМАН Д. И. Этимологический словарь иранских языков. Т. 1-. Москва, 2000-. [RAstorgueva V. S., EdeL'MAN D. I. Etimologicheskij slovar' iranskikh yazykov. T. 1-. Moskva, 2000-.]

ЭССЯ - Этимологический словарь славянских языков. Под ред. О. Н. ТруБАчевА. Т. 1-. Москва, 1974-. [Еtimologicheskij slovar' slavyanskikh yazykov. Pod red. O. N. TrubacheVA. T. 1-. Moskva, 1974-.] 
А. В. ДыБО. Язык и археология: некоторые методологические проблемы. 1. Праиндоевропейская и праалтайская ландшафтная терминология.

Статья представляет собой первую часть работы, в которой проводится попытка систематизировать наши представления о природном окружении и материальной культуре праиндоевропейцев на основании, во-первых, максимально полной выборки реконструированной лексики соответствующих семантических областей, во-вторых, ее сопоставления с такой же выборкой, сделанной для праязыка сходной временной глубины, носители которого явно обитали на территории, не контактной с индоевропейской прародиной - для праалтайского. Здесь представлена лексика, связанная с ландшафтом. Основной вывод заключается в том, что из двух рассмотренных пралексиконов на степное природное окружение указывает скорее праалтайский; праиндоевропейский указывает скорее на горную местность. Что касается водных объектов, для праиндоевропейского окружения следует предполагать наличие моря (или очень большого озера), а для праалтайского - наличие очень больших рек с сезонными разливами.

Ключевые слова: индоевропейская прародина, алтайская прародина, метод слов и вещей, семантическая реконструкция. 